\title{
The Neostriatal Mosaic: II. Patch- and Matrix-Directed Mesostriatal Dopaminergic and Non-Dopaminergic Systems
}

\author{
Charles R. Gerfen, ${ }^{1}$ Miles Herkenham, ${ }^{1, a}$ and Jean Thibault ${ }^{2}$ \\ 'Laboratory of Neurophysiology, National Institute of Mental Health, Bethesda, Maryland 20892, and 'Department of \\ Cellular Biochemistry, Collège de France, Paris
}

\begin{abstract}
Mesostriatal projections, which arise from dopaminergic and non-dopaminergic neurons in the ventral tegmental area, substantia nigra, and retrorubral area, are compartmentally organized in the striatum. Anterograde axonal tract tracing with Phaseolus vulgaris-leucoagglutinin (PHA-L), combined with immunohistochemical localization of tyrosine hydroxylase (TH) and autoradiographic localization of $\mu$-opiate receptor binding sites, shows that midbrain projections to the striatum are distributed to either the $\mu$-opiate receptor-rich "patch" or the receptor-poor "matrix" striatal compartments. Three morphologically distinct mesostriatal afferent fiber types are labeled. The first type, type A, forms a plexus of relatively thin $(0.1-0.4 \mu \mathrm{m})$, smooth fibers with small varicosities (0.3-0.6 $\mu \mathrm{m})$. A second type, type $B$, is similar to the first in forming a plexus of fibers, but is slightly thicker $(0.2-0.6 \mu \mathrm{m})$, with more frequent varicosities $(0.4-1.0 \mu \mathrm{m})$ that give this fiber type a crinkled appearance. The third type, type $C$, constitutes a minority of striatal afferents and is characterized by its large caliber $(0.4-0.7 \mu \mathrm{m})$ with large bulbous varicosities (1.2-2.0 $\mu \mathrm{m})$. Projections of the ventral tegmental area (A10 cell group) are primarily dopaminergic type A fibers directed to the matrix of the ventromedial striatum, including the nucleus accumbens. The retrorubral area (A8 cell group) also provides predominantly dopaminergic type $A$ fibers to the striatal matrix, but these are distributed dorsally. The substantia nigra contains a mixed population of neurons that project to the striatum. Some, located in the dorsal tier of the pars compacta (dorsal A9 cell group), provide dopaminergic type A fibers to the striatal matrix. Others, in the ventral tier of the pars compacta (ventral A9 cell group) and in the ventral tier of the pars reticulata (displaced A9 cells), provide dopaminergic type $B$ fibers to the striatal patches. An additional set of substantia nigra neurons that are non-dopaminergic is the source of type $C$ fibers to the striatal matrix. Thus, distinct dorsal and ventral sets of midbrain dopaminergic neurons project, respectively, to striatal matrix and patches, and there is a non-dopaminergic mesostriatal projection to the matrix.
\end{abstract}

\footnotetext{
Received Dec. 3, 1986; revised Mar. 27, 1987; accepted June 5, 1987

This paper is dedicated to the memory of Dr. Edward V. Evarts, Chief, Laboratory of Neurophysiology, NIMH.

Correspondence should be addressed to C. R. Gerfen, Lab of Cell Biology, NIMH, Bldg. 36, Rm 3A-17, Bethesda, MD 20892.

a Present address: Unit on Functional Neuroanatomy, Biological Psychiatry Branch, NIMH, Bethesda, MD.

0270-6474/87/123915-20\$02.00/0
}

Many aspects of behavior are now thought to be influenced by neuronal activity in the basal ganglia (see DeLong and Georgopoulos, 1981, and Alexander et al., 1986, for review). The striatum is the core nucleus of the basal ganglia, and as many as $95 \%$ of its neurons are of one type, the medium spiny type (Kemp and Powell, 1971). The striatal medium spiny neuron is the major target of extrinsic afferents from the cortex (Kemp and Powell, 1971; Somogyi et al., 1981a) and from the substantia nigra (Freund et al., 1984), and it is the source of the projections to the output nuclei of the basal ganglia - the globus pallidus (Chang et al., 1981) and the substantia nigra (Grofova, 1975; Somogyi and Smith, 1979). Thus, patterns of afferent and efferent connectivity of these striatal neurons must play a crucial role in basal ganglia function. The purpose of the present series of studies is to examine more closely the segregation of striatal medium spiny neurons into either "patch" or "matrix" striatal compartments (Gerfen, 1985), which have been defined on the basis of biochemical markers (Pert et al., 1976; Graybiel and Ragsdale, 1978; Herkenham and Pert, 1981).

Previous work has established the following patterns of connectivity: (1) The efferent targets of patch and matrix neurons are distinct; neurons in the patches project to the substantia nigra pars compacta, whereas neurons in the matrix project to the substantia nigra pars reticulata (Gerfen, 1984, 1985; Gerfen et al., 1985). (2) The dendritic arbors of patch and matrix medium spiny neurons are restricted to their respective compartments (Gerfen, 1984, 1985; Herkenham et al., 1984; Penny et al., 1984; Izzo et al., 1987). (3) Local collaterals of these ncurons share the same domain as do their dendrites (Wilson and Groves, 1980; Somogyi et al., 1981a; Bishop et al., 1982). (4) Cortical and thalamic afferents are compartmentally restricted (Herkenham and Pert, 1981; Ragsdale and Graybiel, 1981; GoldmanRakic, 1982; Beckstead, 1984; Gerfen, 1984; Donoghue and Herkenham, 1986). Taken together, the above findings suggest that the patch and matrix compartments are segregated, parallel input-output systems (Gerfen, 1984). The present study examines the question of whether separate dopamine systems exist with respect to striatal compartmentalization.

The mesostriatal dopaminergic system exerts a profound influence on the striatum, affecting both motor and motivational aspects of behavior (Hornykiewicz, 1966; Stevens, 1973; Penney and Young, 1983; Fibiger and Phillips, 1986). It has been proposed that motor and motivational functions of the striatum may be carried out by the dorsal and ventral striatum, respectively (Heimer and Wilson, 1975; Nauta et al., 1978; Kelley et al., 1982). More recently, we have postulated that the patchmatrix compartments may provide an additional organization 
by which limbic cortical inputs may be conveyed to patches in the dorsal striatum (Gerfen, 1984; Donoghue and Herkenham, 1986). With regard to possible segregation and/or compartmentalization of putative mesolimbic dopaminergic inputs (from the A 10 cell group in the ventral tegmental area) and non-limbic inputs (presumably from the A9 cell group in the substantia nigra pars compacta and perhaps also from the A8 cell group in the retrorubral area), previous studies have shown that the ventral tegmental area projects preferentially to the striatal matrix (Herkenham et al., 1984), whereas the substantia nigra projects to both compartments, but more densely to the patches (Wright and Arbuthnott, 1981; Moon-Edley and Herkenham, 1984). A similar organization is observed in cats (Beckstead, 1984). While such studies attempted to correlate the compartmental organization in terms of the classic parcellation of the A10, A9, and A8 dopamine cell groups (Dahlström and Fuxe, 1964), other evidence (Gerfen, 1986) suggested that segregation of mesostriatal neurons into dorsal and ventral tier cell groups (Fallon and Moore, 1978; Veening et al., 1980) most likely represented the separate origins of the patch- and matrix-directed systems.

In the present studies, the projections of the mesostriatal system are reexamined with the Phaseolus vulgaris-leucoagglutinin (PHA-L) anterograde and axonal tract-tracing technique (Gerfen and Sawchenko, 1984). Dopaminergic striatal afferent axons are distinguished by the presence or absence of immunohistochemically colocalized PHA-L and tyrosine hydroxylase $(\mathrm{TH})$ (Gerfen and Sawchenko, 1985). The relationship of these inputs to the striatal patch and matrix compartments is examined by comparing the distribution of PHA-L-labeled striatal afferents from various sites in the ventral midbrain, using ${ }^{3} \mathrm{H}$-naloxone binding to opiate receptors as a marker of the striatal patches.

\section{Materials and Methods}

Adult female albino rats (OM strain) were used in this study. Animals were anesthetized with chloral hydrate, their heads immobilized in a Kopf small animal stereotaxic apparatus, and axonally transported tracers were injected into their brains, as described below. Following a $10 \mathrm{~d}$ postoperative period, animals were anesthetized with an overdose of chloral hydrate and, under deep anesthesia, perfused transcardially with $150 \mathrm{ml}$ ice-cold $150 \mathrm{~mm} \mathrm{NaCl}$, followed by $500 \mathrm{ml}$ fixative solution (room temperature) that contained $4 \%$ formaldehyde (from paraformaldehyde), $1 \%$ calcium acetate, $20 \mathrm{~mm}$ sodium phosphate buffer ( $\mathrm{pH}$ 7.4), and $100 \mathrm{~mm} \mathrm{NaCl}$. The brains were removed, postfixed in the perfusion fixative for $24-48 \mathrm{hr}$, and transferred to a solution of $150 \mathrm{~mm}$ $\mathrm{NaCl}$ and $20 \%$ sucrose for an additional $18 \mathrm{hr}$. Brains were cut frozen into $30-\mu \mathrm{m}$-thick sections that were collected in $20 \mathrm{~mm}$ potassium phosphate-buffered $150 \mathrm{~mm}$ saline (KPBS, $\mathrm{pH} 7.4$ ) and then processed according to the procedures described in detail below.

Retrograde axonal tracing studies. The neurochemical phenotype of mesostriatal neurons was determined by the method of Sawchenko and Swanson (1981) for immunohistochemically labeling neurons that are also fluorescently labeled with a retrograde axonal tracer. Multiple 2.0 $\mu l$ injections of a $3 \%$ solution of the retrogradely transported fluorescent dye fast bluc (Bentivoglio et al., 1980) werc made into the striatum. Following the protocol outlined above, sections through the striatum were mounted to determine the sites of dye injection. Sections through the midbrain were incubated in a solution of rabbit antiserum directed against tyrosine hydroxylase (R $\alpha \mathrm{TH}$; Arluison et al., 1984) diluted 1:1500 in KPBS plus $0.5 \%$ Triton X-100 and $2 \%$ normal goat serum (NGS). Following a $24-48 \mathrm{hr}$ incubation, sections were rinsed and then incubated in a dilution of antiserum directed against rabbit $\mathrm{IgG}$, labeled with either fluorescein (G $\alpha$ R-FITC) or tetramethylrhodamine (G $\alpha$ RTRITC) diluted $1: 200$ in KPBS plus 2\% NGS and $0.5 \%$ Triton X-100 for 45-60 min. Sections were then rinsed, mounted onto gelatin-coated slides, air-dried, and coverslipped with buffered glycerol mountant $(\mathrm{pH}$ 8.5). Labeled neurons were identified by epifluorescent illumination, using filters to specifically activate one fluorochrome, as follows: fast blue-Nikon Filter UV (excitation filter $330-380 \mathrm{~nm} /$ barrier filter 420 $\mathrm{nm}$ ); FITC-modified Nikon Filter B1 (excitation filter 460-485 nm/ barrier filter $515-545 \mathrm{~nm}$ ); rhodamine-Nikon filter $\mathrm{G}$ (excitation filter $535-550 \mathrm{~nm} /$ barrier filter $580 \mathrm{~nm}$ ).

PHA-L axonal tract tracing. The PHA-L anterograde axonal tracttracing method (Gerfen and Sawchenko, 1984) was used to examine the projections from the midbrain to the striatum. Adjacent sections through the forebrain were separated into 3 series, each of which was processed by one of the following methods: (1) standard immunoperoxidase procedures to localize axonally transported PHA-L, (2) a modification (Gerfen et al., 1985) of the procedure of Herkenham and Pert (1982) to localize $\mu$-opiate receptors by in vitro autoradiography, and (3) allowing neurochemical identification of PHA-L-labeled axons (Gerfen and Sawchenko, 1985). The standard PHA-L method for tracing efferent axonal connections was followed. A $2.5 \%$ solution of PHA-L (Vector Laboratories) in $20 \mathrm{~mm}$ sodium phosphate-buffered $150 \mathrm{~mm}$ saline (NaPBS, $\mathrm{pH}$ 7.4) was loaded into glass micropipettes with tip diameters of 10 $15 \mu \mathrm{m}$. With the rats anesthetized with chloral hydrate, injections of PHA-L were made into the ventral midbrain by ionotophoresis using $7 \mu \mathrm{A}$ positive current pulsed every other $7 \mathrm{sec}$ for $20 \mathrm{~min}$ with a constant current device (CS-3; Transkinetics, Compton, MA). Following the postoperative survival period (10-21 d), perfusion-fixation and tissue-processing procedures, as described above, were followed. Sections through the midbrain were processed to determine the site of the PHA-L injection. Three series of adjacent sections through the forebrain were processed.

PHA-L axonal labeling. The first series was processed to localize axonally transported PHA-L using an immunoperoxidase method. Sections were incubated in a rabbit antiserum directed against PHA-L (diluted 1:2000 in KPBS plus $2 \%$ NGS and $0.5 \%$ Triton X-100). Following an incubation at $4^{\circ} \mathrm{C}$ for $24-48 \mathrm{hr}$, sections were rinsed and reacted with the avidin-biotin immunoperoxidase method (Hsu et al., 1981; Vector Laboratories, Burlingame, CA). Sections were incubated for $60 \mathrm{~min}$ in a solution containing biotin-labcled goat antiserum directed against rabbit IgG (44 $\mu 1$ in $10 \mathrm{ml} \mathrm{KBPS}$ plus $2 \%$ NGS and $0.5 \%$ Triton $\mathrm{X}-100$ ). Sections were then rinsed and incubated in a premixed solution containing $88 \mu \mathrm{l} / 10 \mathrm{ml}$ each of the components of the avidinbiotin-peroxidase complex (ABC) in KPBS for $60 \mathrm{~min}$ and then rinsed. Peroxidase localization was obtained by incubating the sections in a filtered solution of KPBS containing $0.05 \%$ diaminobinzidine (DAB; Organon Technica) and $0.005 \%$ hydrogen peroxide for $10-20 \mathrm{~min}$. To stop the reactions, sections were transferred to $10 \%$ formalin for 5-15 min, rinsed in KPBS, and mounted onto gelatin-coated slides. Slidemounted sections were then defatted twice for $30 \mathrm{~min}$ in chloroform/ methanol, rehydrated by passage through descending concentrations of alcohol solutions, and intensified in a solution of $0.001 \%$ osmium tetroxide for 2-4 hr, rinsed, dehydrated, and coverslipped out of xylene.

${ }^{3} H$-Naloxone labeling of striatal compartments. The sccond scrics of sections was processed to demonstrate in vitro ${ }^{3} \mathrm{H}$-naloxone binding to the $\mu$-opiate receptor-rich striatal patch compartment for a comparison of the compartmental distributions of PHA-L-labeled afferents in adjacent sections. Except for the initial perfusion fixation of the animal and freezing microtome sectioning of the brain, the procedure described by Herkenham and Pert (1982) for the autoradiographic localization of receptor binding was followed. Following sectioning, sections were collected in ice-cold KPBS and mounted within $6 \mathrm{hr}$ of cutting onto gelatincoated slides, air-dried, and stored at $-20^{\circ} \mathrm{C}$ until further processing. Slide-mounted sections were incubated in a solution containing $2.5 \mathrm{nM}$ ${ }^{3} \mathrm{H}$-naloxone (sp act, $44.4 \mathrm{Ci} / \mathrm{mmol}$; New England Nuclear) in $50 \mathrm{~mm}$ Tris buffer (pH 7.4) and $100 \mathrm{~mm} \mathrm{NaCl}$ at $4^{\circ} \mathrm{C}$ for $90 \mathrm{~min}$, and then rinsed 3 times for $1 \mathrm{~min}$ in solutions of Tris-buffered saline, air-dried, fixed in formaldehyde vapors $\left(80^{\circ} \mathrm{C}\right)$ for $90 \mathrm{~min}$, dehydrated, defatted in chloroform $/$ methanol, rehydrated, and air-dried. Sections were then dipped in Kodak NTB-2 nuclear track emulsion, dried, and exposed for 3 months, after which they were developed in Kodak D-19 developer $\left(2 \mathrm{~min}\right.$ at $17^{\circ} \mathrm{C}$ ), stopped, fixed, and rinsed. After counterstaining through the emulsion with thionin, sections were coverslipped out of xylene.

Tyrosine hydroxylase immunoreactivity in PHA-L-labeled fibers. To distinguish presumptive dopaminergic and non-dopaminergic nigrostriatal afferents, a modification of the PHA-L method that allows chemical determination of labeled fiber tracts was used (Gerfen and Sawchenko, 1985). Sections from the striatum and midbrain were incubated in a mixture of $\mathrm{R} \alpha \mathrm{TH}(1: 1500)$ and guinea pig antiserum directed against PHA-L (GP $\alpha$ PHA-L, 1:2000) diluted in KPBS plus 2\% NGS and $0.5 \%$ 
Triton X-100 for $48 \mathrm{hr}$ at $4^{\circ} \mathrm{C}$. Sections were then rinsed and incubated in a mixture of fluorochrome-labeled secondary antisera that included rhodamine-conjugated goat antibody directed against guinea pig IgG (G $\alpha$ GP-TRITC, 1:200; Cappel Labs) and fluorescein-conjugated $\mathrm{G} \alpha \mathrm{R}$ FITC (1:200; Sigma Chemical Co.) diluted in KPBS plus $2 \%$ NGS and $0.5 \%$ Triton X-100 for $45-60 \mathrm{~min}$ at room temperature. Sections were then rinsed, mounted out of KPBS onto cirrom-alum-coated slides, airdried, and coverslipped with buffered glycerol $(\mathrm{pH} 8.5)$.

\section{Results}

Distribution of ventral midbrain neurons projecting to the striatum

TH immunofluorescent labeling and retrograde axonal labeling with the fluorescent dye fast blue after injections into the striatum were combined to map the distribution of dopaminergic and non-dopaminergic mesostriatal neurons. In 4 cases the injections were centered within the striatum, including the nucleus accumbens, and there appeared to be little or no diffusion of the dye outside the boundaries of the striatum to adjacent cortical or olfactory areas. However, it is not assumed that spread of the dye had not occurred to these extrastriatal sites, as during the $10 \mathrm{~d}$ survival period there may have been some decrease in the actual spread of the tracer. Additionally, with fluorescent retrogradely transported dyes there is always the problem of the uptake of the tracer by fibers passing through, but not terminating in, the area of injection. Because of these severe limitations, these cases are used solely for the purpose of mapping the distribution of midbrain neurons that may provide inputs to the striatum, and to determine the location of non-dopaminergic neurons contributing to this projection.

Figure 1 shows photomicrographs of 3 coronal levels from rostral to caudal through the ventral midbrain with TH-immunoreactive neurons labeled with rhodamine (Fig. 1, $A-C$ ) and the same sections viewed with a fluorescent filter to show fast blue-labeled mesostriatal neurons (Fig. 1, $A^{\prime}-C^{\prime}$ ). From the 4 cases, 2 sections from each level shown in Figure 1 were examined at $200 \times$ and the total numbers of retrogradely labeled neurons in these sections were counted to determine the percentage that expressed $\mathrm{TH}$ immunoreactivity. In the cases examined, an average of $85 \%$ of the fast blue neurons labeled expressed TH immunoreactivity. Of the $15 \%$ TH-negative mesostriatal neurons, some $13 \%$ were located in the ventral tegmental area, 20-26\% were in the substantia nigra pars compacta, intermixed with TH-positive neurons, $26-33 \%$ were in the mediodorsal pars reticulata, often abutting the ventral aspect of the pars compacta, and $26 \%$ were in the ventrolateral tier of the pars reticulata, intermixed with dopaminergic mesostriatal neurons in this region.

Dopaminergic neurons (i.e., neurons expressing TH immunoreactivity) that are labeled by striatal injections of fast blue have a widespread distribution. For the purposes of this study, several features of the distributions of these mesostriatal cells are emphasized. First, the 3 dopaminergic cell groups, the A10 cell group in the ventral tegmental area, the A9 cell group in the substantia nigra, and the A8 cell group in the retrorubral area, while regionally localized, appear to form a continuous cell group. Second, the dopaminergic A9 cell group in the substantia nigra is heterogeneous. In the rostral quarter of the nucleus, these cells are confined to the pars compacta dorsal to the pars reticulata. Some of these neurons, in the ventral part of the pars compacta, have dendrites extending ventrally into the pars reticulata (Fig. 1A). Caudal to this, dopaminergic neurons are distributed in both the pars compacta and in the ventral parts of the pars reticulata (Fig. $1 B$ ). It should be noted that the designation of the pars compacta and pars reticulata subdivisions of the substantia nigra predated the identification of dopaminergic neurons. Unfortunately, the terms "pars compacta" and "pars reticulata" have become synonomous with the dopaminergic and non-dopaminergic cell groups, respectively. In the present study these ventrally positioned neurons are referred to as dopaminergic cells in the pars reticulata. The numbers of such dopaminergic neurons increase caudally until, at the most caudal level, there are large numbers of dopaminergic neurons in the pars reticulata (Fig. 1, C, $C^{\prime}$ ).

\section{Morphology of mesostriatal afferents: types $A, B$, and $C$}

PHA-L injections into the ventral midbrain label at least 3 morphologically distinct types of striatal afferents, designated here as types A, B, and C (Fig. 2). Type A is the thinnest, and type $C$ the thickest. Both type $A$ and type $B$ fibers are distributed in dense plexuses, and they possess varicosities that are only slightly larger than the fibers themselves. However, the diameters of the 2 fiber types differ: type A fibers and varicosities are $0.1-0.4$ and $0.3-0.6 \mu \mathrm{m}$, respectively, whereas type B fibers and varicosities are $0.2-0.6$ and $0.4-1.0 \mu \mathrm{m}$, respectively. Moreover, type A fibers are relatively smooth, whereas type B fibers have a more wrinkled or crinkled aspect, owing, possibly, to the more frequent varicosities distributed along them. Also, type A fibers are distributed in the matrix, whereas type B fibers are restricted to the patch compartment (see below). Both type A and type $B$ fibers can be found to branch from much thicker fibers (1.0 $\mu \mathrm{m}$ diameter).

Type $C$ fibers are morphologically distinct from types $A$ and B. Although they also arise from thick fibers, type $C$ fibers retain a grealer thickness $(0.4-0.7 \mu \mathrm{m})$ until they branch and terminate with large, bulbous boutons (1.2-2.0 $\mu \mathrm{m}$ diameter). Type $\mathrm{C}$ fibers are much less frequently labeled than the other fiber types, and they are more often labeled after injections into the pars reticulata than into the pars compacta. Though sparse in number, they are distributed throughout the striatum (see Fig. $9 B$ ).

\section{Neurochemical identification of striatal afferents}

Examination of TH-immunoreactive fibers in the striatum shows them to have the morphology of the PHA-L-labeled type A and B fibers. Large bulbous terminals characteristic of PHA-L-labeled type $\mathrm{C}$ fibers are only rarely labeled with TH immunoreactivity. The occasional $\mathrm{TH}$-positive type $\mathrm{C}$ fibers are most often found in the ventralmost striatum and appear similar in morphology to TH-immunoreactive fibers in the olfactory tubercle. The density of TH-labeled fibers makes pholography of individual fibers difficult. To more clearly document these fibers, low doses of 6-hydroxydopamine $(1 \mu \mathrm{g}$ in $1 \mu \mathrm{l})$ were injected into the medial forebrain bundle of 3 rats, which were then allowed to survive for 6 weeks. Such treatment resulted in only a partial denervation of midbrain dopaminergic neurons, and also in a "thinning-out" of TH-immunoreactive fibers in the striatum. Surviving TH-immunoreactive fibers in the striatum were identical morphologically, in terms of their fiber thickness and varicose appearance, to those in the unlesioned animal. A photomicrograph of TH-immunoreactive fibers from such a case is shown in Figure $2 D$. These fibers have the morphologies of both PHA-L-labeled type A or B fibers. Large-diameter TH- 

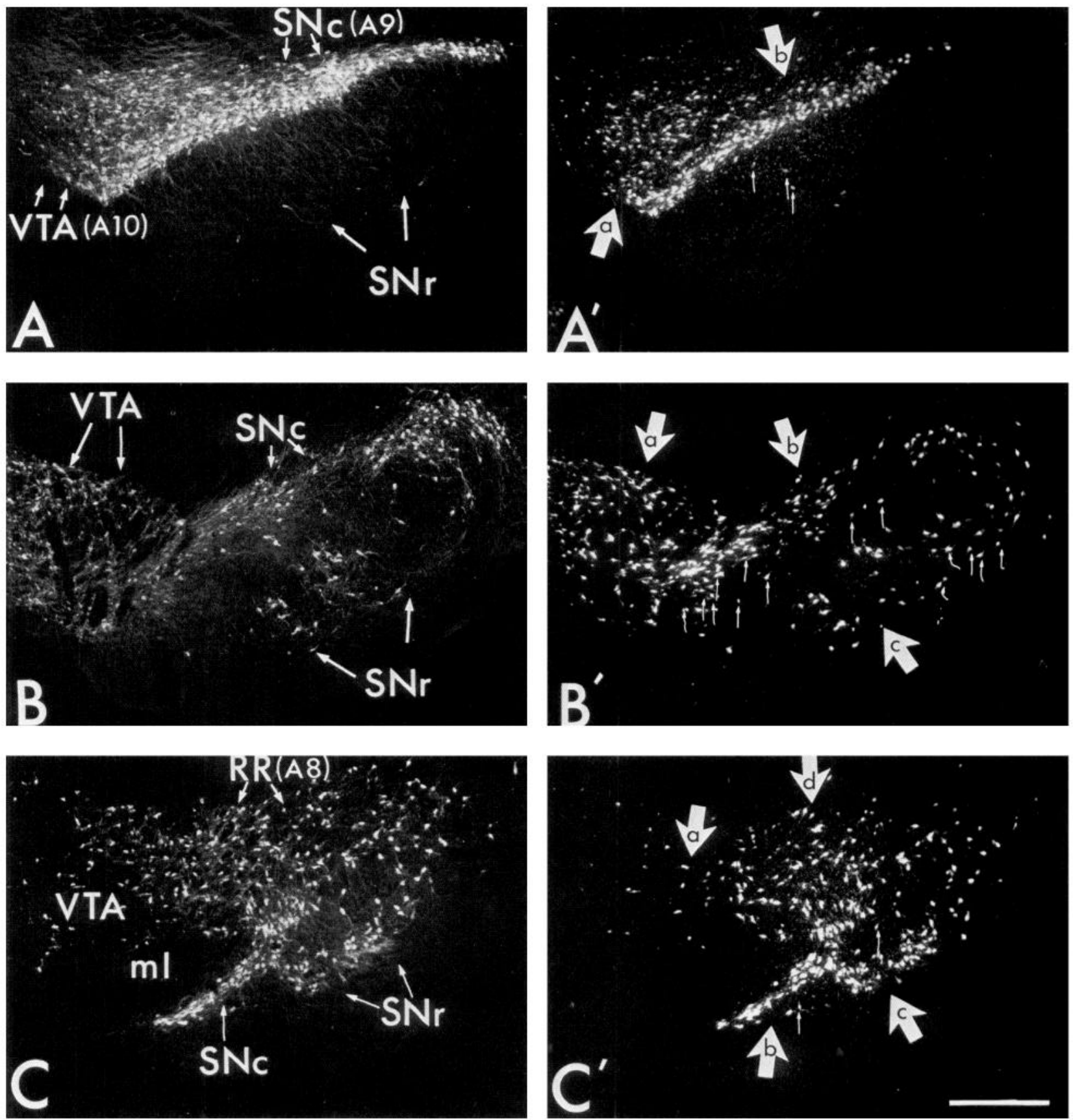

Figure 1. Coronal sections through rostral $(A)$, mid $(B)$, and caudal $(C)$ levels of the ventral midbrain, photographed with separate fluorescent filters to show the distribution of dopaminergic neurons $(A-C)$ labeled with tyrosine hydroxylase (TH) immunoreactivity and mesostriatal neurons $\left(A^{\prime}-C^{\prime}\right)$ labeled by retrograde transport of fast blue from injections into the striatum. The location of the A10, A9, and A8 dopamine cell groups are marked. The A10 cell group is located medially at all 3 levels in the ventral tegmental area $(V T A)$. At rostral levels $(A)$, the A9 cells are confined to the substantia nigra pars compacta $(S N c)$. The ventrally directed dendrites extending into the pars reticulata $(S N r)$ of some of these neurons are evident. At mid-rostrocaudal levels $(B)$, A9 dopaminergic neurons are located in both the $\mathrm{SNc}$ and ventral $\mathrm{SNr}$. At caudal levels $(C)$ there are numerous A9 cells in the $\mathrm{SNc}$ and $\mathrm{SNr}$ and $\mathrm{A} 8$ cells are located dorsally in the retrorubral area $(R R)$. The majority $(85 \%)$ of retrogradely labeled neurons $\left(A^{\prime}-C^{\prime}\right)$ are TH-immunoreactive $(A-C)$. Such doubly labeled cells are localized in the VTA (arrow $\left.a, A^{\prime}-C^{\prime}\right), \mathrm{SNc}\left(a r r o w b, A^{\prime}-C^{\prime}\right), \mathrm{SNr}$ (arrow $c, B^{\prime}$ and $C^{\prime}$ ), and $R R$ (arrow $\left.d, C^{\prime}\right)$. Non-dopaminergic neurons are localized in the VTA, SNc, medial SNr $\left(A^{\prime}, B^{\prime}\right.$, small arrows) and ventrolateral $\mathrm{SNr}\left(B^{\prime}\right.$, small arrows $)$. Scale bar, $500 \mu \mathrm{m}$.

immunoreactive fibers are observed, but these most often branch into fine-caliber type A or type B fibers.

By combining immunofluorescent localization of TH immunoreactivity with PHA-L labeling of striatal afferents, it is possible to determine which of type A, B, and C fiber types are presumed to be dopaminergic. The methodology and requisite controls for this procedure have been described previously (Gerfen and Sawchenko, 1985). Figure 3, $A, A^{\prime}$ shows an injection of PHA-L into the substantia nigra pars compacta and pars reticulata, which labels both TH-positive and TH-negative neurons. Representatives of each of the 3 striatal afferent fiber types are labeled by this injection. PHA-L-labeled fibers of thin cal- 

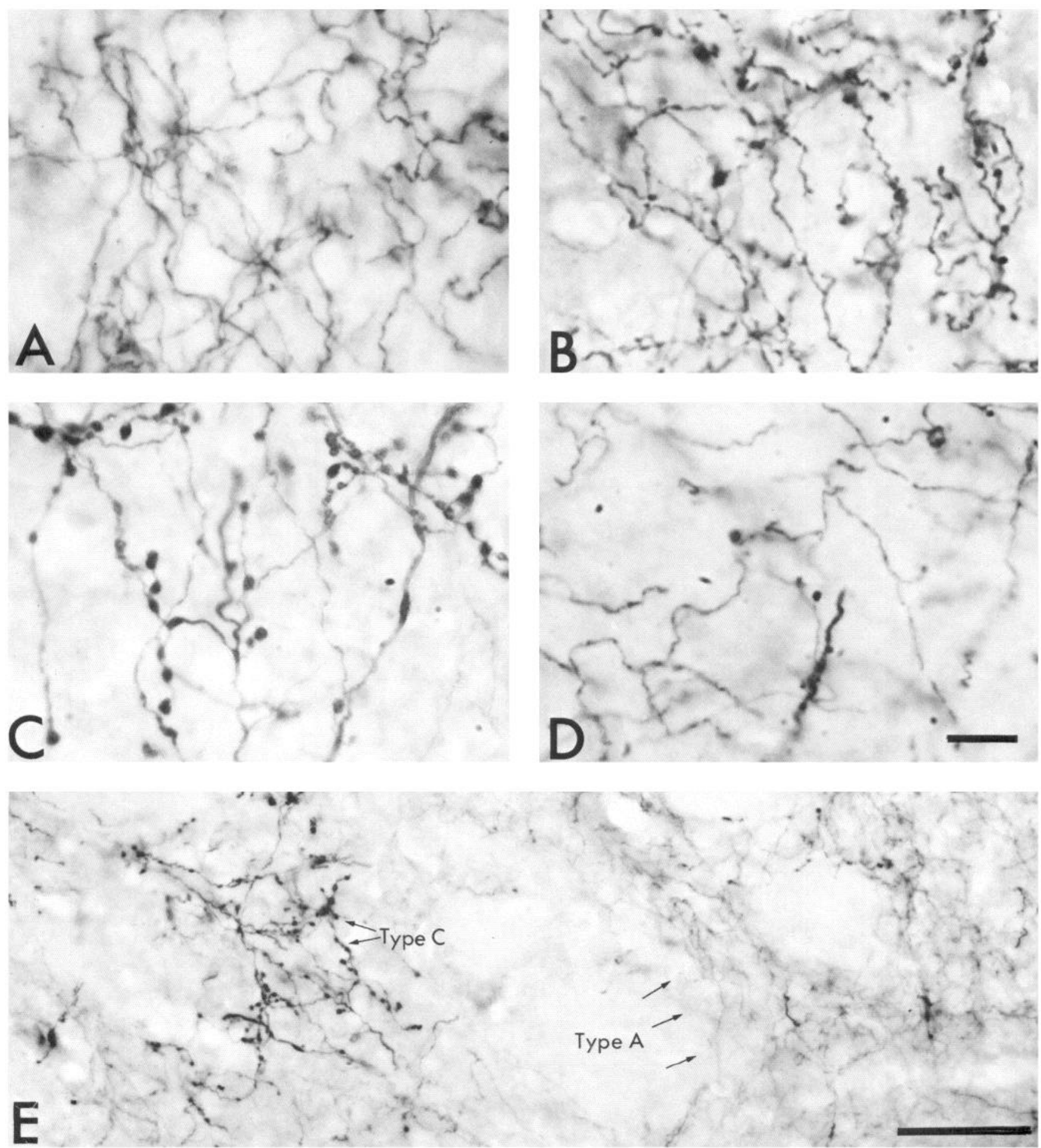

Figure 2. Three morphologically distinct striatal afferent fibers labeled with PHA-L injections into different regions of the ventral midbrain are shown. Type A fibers $(A)$ are relatively thin $(0.1-0.4 \mu \mathrm{m})$ with slight swellings $(0.4-0.6 \mu \mathrm{m})$, and form a dense plexus. These fibers are distributed in the striatal matrix compartment. Type B fibers $(B)$ are slightly thicker $(0.2-0.6 \mu \mathrm{m})$ than type A fibers, and also have slight swellings $(0.4-1.0$ $\mu \mathrm{m})$. These fibers also form a feltwork and are distinct from type $\mathrm{A}$ in their somewhat flattened, ribbonlike appearance. Type $\mathrm{C}$ fibers $(C)$ are thicker $(0.8 \mu \mathrm{m})$ than either type A or B fibers, and branch in distinct arbors with large bulbous swellings $(1.2-2.0 \mu \mathrm{m}) . D$, TH-immunoreactive fibers from a case in which low doses of 6-OHDA were injected into the medial forebrain bundle to thin out striatal dopaminergic fibers. The THimmunoreactive fibers in $D$ are similar in morphological appearance to those of type A PHA-L-labeled mesostriatal fibers. E, A low-power photomicrograph shows an area of the striatum in which both type $\mathrm{C}$ (on the left) and type A (on the right) PHA-L-labeled nigrostriatal afferents are labeled. Scale bar, $10 \mu \mathrm{m}(A-D)$ and $100 \mu \mathrm{m}(E)$. 

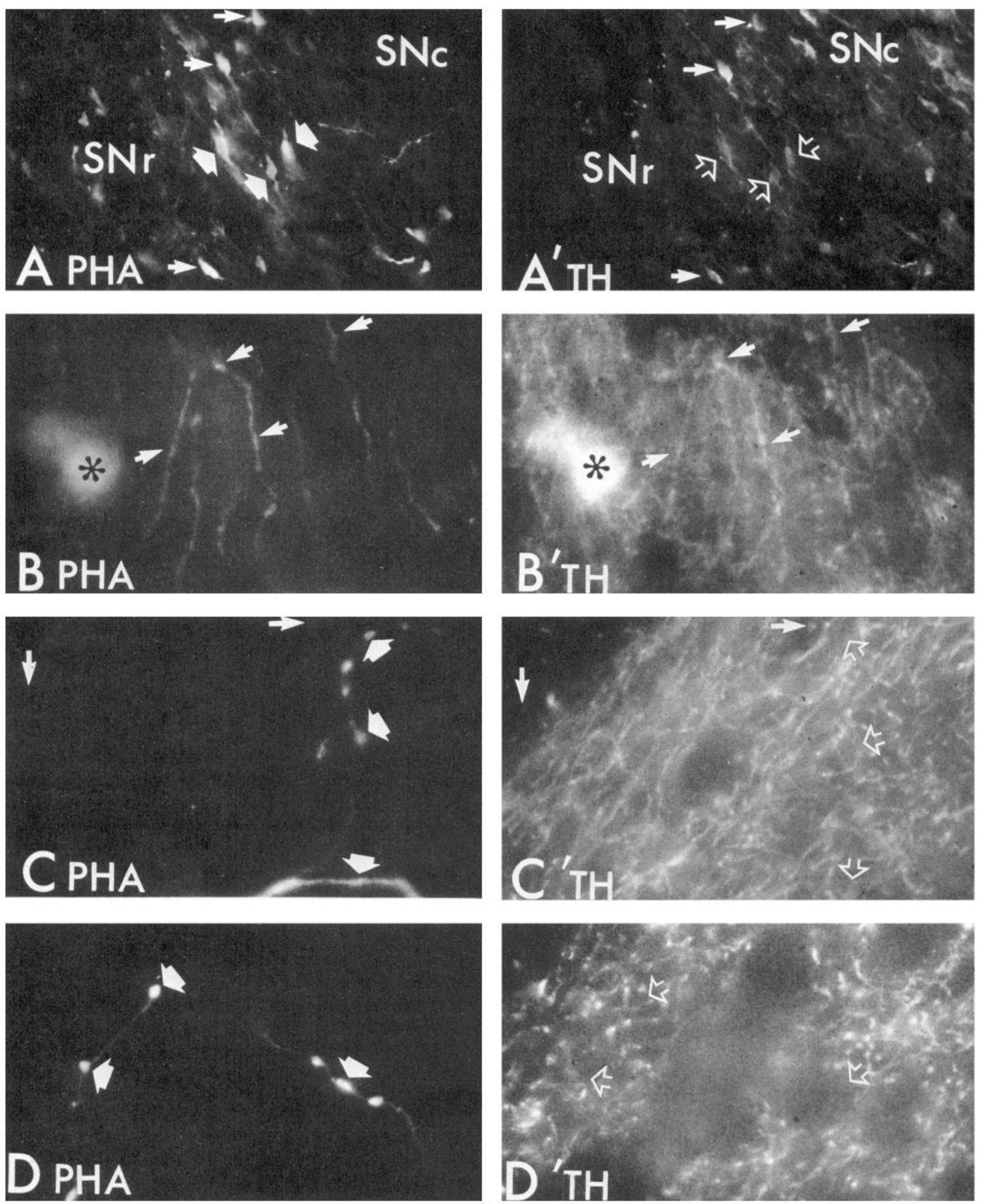

Figure 3. Colocalization in the same sections of PHA-L and TH immunoreactivity was obtained by photographing a section with a rhodaminespecific fluorescence filter to identify PHA-L immunoreactivity labeled with GP $\alpha$ PHA-L-G $\alpha$ GP-TRITC $(A-C)$ and then with a fluorescein-specific fluorescence filter to identify TH immunoreactivity labeled with R $\alpha \mathrm{TH}-\mathrm{G} \alpha \mathrm{R}-\mathrm{FITC}\left(A^{\prime}-C^{\prime}\right)$. In $A$, PHA-L-labeled neurons at the site of injection into the substantia nigra pars compacta (SNc) are shown in $A^{\prime}$ to be either TH-positive (solid arrows) or TH-negative (open arrows). This injection labeled both dopaminergic and non-dopaminergic nigrostriatal neurons. The substantia nigra pars reticulata (SNr) is at left. In $B$, PHA-L-labeled nigrostriatal type $\mathrm{A}$ afferents are shown in $B^{\prime}$ to be TH-positive and are thus presumed to be dopaminergic. In $C$ and $D$, PHA-L-labeled nigrostriatal afferents of type $C$, with distinctive large bulbous boutons, are shown in $C^{\prime}$ and $D^{\prime}$ to be TH-negative and are thus presumed to be non-dopaminergic. Broad solid arrows point to the location of either PHA-L-labeled boutons or fibers labeled in $C$ and $D$ that are not labeled with TH immunoreactivity 
iber, both type $\mathrm{A}$ and type $\mathrm{B}$, are colabeled with $\mathrm{TH}$ immunoreactivity. Examples of type A-labeled fibers are shown in Figures $3, B, B^{\prime}, 4, A, A^{\prime}$, and type B-labeled fibers are shown in Figure $4, B, B^{\prime}$. Some thick labeled fibers from which thinner fibers branched are also colabeled with $\mathrm{TH}$ immunoreactivity. PHA-L-labeled type C striatal afferent fibers in the dorsal striatum have never been shown to contain TH immunoreactivity. The distinct bulbous boutons can be clearly identified. The thick fibers from which type $\mathrm{C}$ fibers branched are also TH-negative (Figs. 3, $C, C^{\prime} ; 4, C, C^{\prime}$ ). Although the vast majority of type $C$ fibers are TH-negative, because some have been identified as TH-positive, care was taken to discriminate such TH-negative fibers in sections adjacent to those labeled for striatal compartment identification (see below).

\section{Patch-matrix distribution of mesostriatal afferents}

Over 120 cases of PHA-L labeling of mesostriatal afferents were used in this study. In this report, 6 cases that represent projections from subregions of the midbrain are documented. These cases provide the following information: (1) The locations and chemical phenotypes (TH immunoreactive or not) of the injected neurons. (2) Identification of the morphology of the striatal afferent fiber, with its chemical phenotype determined. (3) Detcrmination of both the rcgional distribution within the striatum and the compartmental distribution (patch or matrix) for fibers characterized according to (1) and (2).

Ventral tegmental area. PHA-L injections into the ventral tegmental area label predominantly $\mathrm{TH}$-immunoreactive neurons, while some $15 \%$ are TH-negative. Labeled efferents are distributed to the prefrontal cortex, striatum, septum, amygdala, olfactory tubercle, pyriform cortex, thalamus, and central gray matter. In the thalamus, all PHA-L-labeled fibers are TH-negative, whereas in the forebrain there are both $\mathrm{TH}$-positive and TH-negative fibers labeled with PHA-L. The morphology of labeled afferents in the striatum is predominantly that of fiber type A. Such fibers form a dense plexus in the nucleus accumbens and ventromedial caudate-putamen (see Fig. 8A). As compared to the distribution of ${ }^{3} \mathrm{H}$-naloxone binding to mark striatal patches, afferents labeled from the ventral tegmental area are scen to be distributcd in the surrounding matrix regions and to be relatively sparse in the patches (Fig. $8 A$ ). In the present cases there are few TH-negative striatal afferents labeled after ventral tegmental area injections. Those labeled have fiber type $\mathrm{C}$ morphology and are distributed in the matrix. Additionally, there is an occasional striatal afferent that has large boutons approaching the size of the type $\mathrm{C}$ fibers, which displays $\mathrm{TH}$ immunoreactivity. Such fibers are very rare and have only been identified in the ventral striatum after ventral tegmental area injections. There are also TH-negative PHA-L-labeled afferents with similar morphology in the olfactory tubercle.

Substantia nigra. PHA-L injections provide different types of striatal afferent labeling, dependent on the site of labeled neurons in the substantia nigra. Four cases are detailed as representative. In the first case (Fig. $8 B$ ), the PHA-L injection is in the rostral pars compacta. In this case, 76 cells were identified as bcing labclcd with PHA-L; of thesc, 65 were located in the dorsal tier of the pars compacta and had dendrites that extended mediolaterally in the plane of the nucleus. The other labcled neurons were located in either the ventral part of the pars compacta and had dendrites extending into the pars reticulata or were located in the pars reticulata. In sections colabeled for TH immunoreactivity, 20 of 23 PHA-L-labeled neurons were colabeled. The labeled striatal projections express TH immunoreactivity, are of fiber type $\mathrm{A}$, and are distributed in dorsolateral matrix areas (Figs. $7, A, A^{\prime} ; 8 B$ ). These fibers are distributed throughout the rostrocaudal extent of the striatum (Fig. 11). PHA-L-labeled fibers are also distributed in the olfactory tubercle and pyriform cortex, where most are $\mathrm{TH}$-positive. While there is some labeling of nigrothalamic and nigrotectal fibers, it is relatively sparse compared with labeling of these fibers after injections of PHA-L into the pars reticulata. These labeled efferents are TH-negative.

The second described PHA-L injection is centered in the ventrolateral substantia nigra pars reticulata at a level midway along its rostrocaudal extent. The injection site is shown in Figure $5 D$, and its striatal afferents are shown in Figures $6 B$ and $7 A$ and charted in Figure $8 D$. Dense astrocytic labeling at the injection site makes a clear morphological and biochemical characterization of all the neurons labeled impossible. Labeled efferents include dense projections to the superior colliculus, thalamus, pedunculopontine nucleus, and striatum. Only striatal afferents are TH-positive, and these are primarily of type B and are distributed principally to patches identified with ${ }^{3} \mathrm{H}$-naloxone binding in adjacent sections. Patches with labeled afferents are located in the dorsolateral striatum. There is a small number of TH-negative fibers labeled in the striatum. These are distributed in the matrix and are of fiber type C. As is evident from a comparison of the projections of this case with those described above, both nigral areas provide $\mathrm{TH}$-positive afferents to the same dorsolateral striatal region, but to complementary compartments.

Whereas the previous 2 representative cases showed preferential projections to either the patch or matrix striatal compartments, a third PHA-L injection shows projections distributed to both compartments. The injection shown in Figure $5 \mathrm{C}$ labels neurons in both the dorsal and ventral tiers of the substantia nigra pars compacta. The density of PHA-L labeling of astrocytes precludes a clear delineation of all the morphologic features of such neurons. However, it is possible to distinguish labeled dendrites that extend into the pars reticulata, as well as those that extend mediolaterally in the plane of the pars compacta. With double-immunofluorescence methods it is evident that both $\mathrm{TH}$-positive and negative neurons are labeled (Fig. 3, $\left.A, A^{\prime}\right)$. Nigrotectal and nigrothalamic efferents are labeled and are TH-negative. Labeled striatal afferents are distributed in the dorsal striatum (Fig. $8 \mathrm{C}$ ) and most are TH-positive, although a few of fiber type $\mathrm{C}$ are not. As is evident in Figures $6 \mathrm{~A}$ and $7 C$, both patch and matrix compartments contain labeled afferents. The labeling in patches is of type B fibers, which appears qualitatively denser than the labeling in the matrix. The vast majority of labeled fibers in the matrix are TH-positive and type A.

A fourth PHA-L injection is centered in the medial pars reticulata and labels few, if any, pars compacta neurons. Labeled

in $C^{\prime}$ and $D^{\prime}$ (open arrows), respectively. In $C$, some PHA-L-labeled type A fibers (solid arrows) are seen in $C^{\prime}$ to express TH immunoreactivity (solid arrows). 

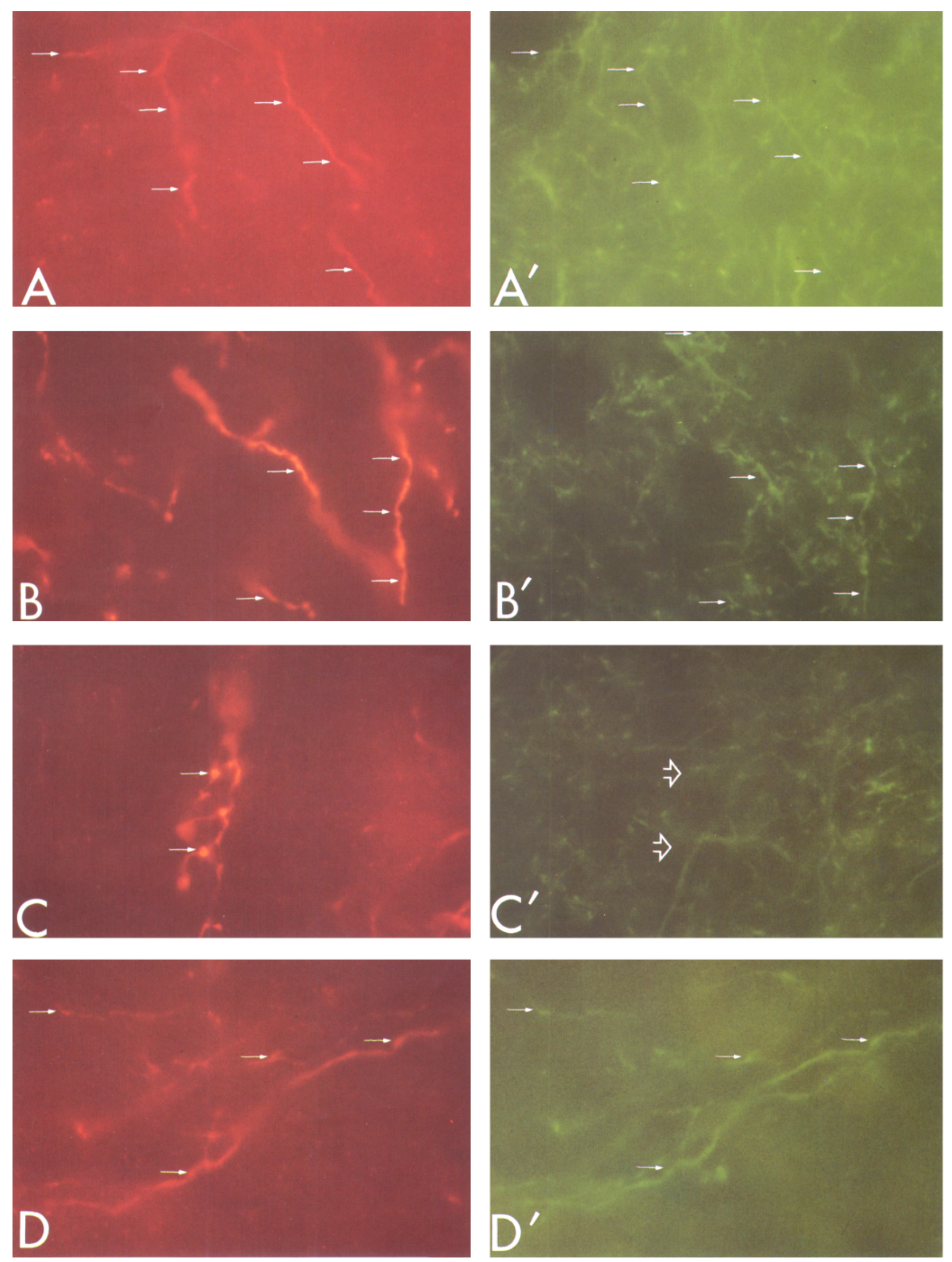

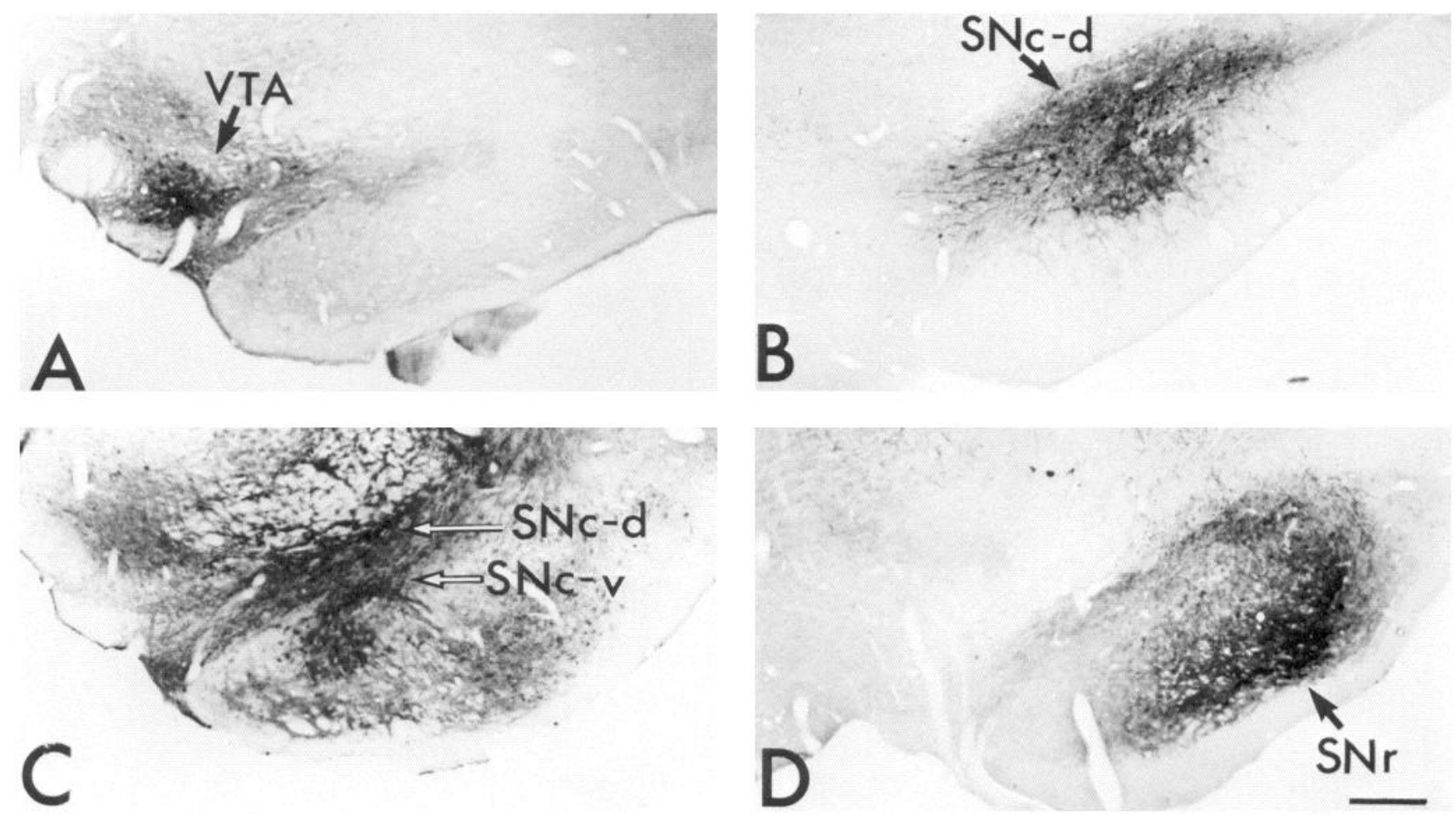

Figure 5. Photographs of PHA-L injections into $(A)$ the ventral tegmental area $(V T A) ;(B)$ the dorsal tier of the substantia nigra pars compacta $(S N c-d) ;(C)$ the dorsal $(S N c-d)$ and ventral $(S N c-v)$ tier of the substantia nigra pars compacta; and $(D)$ the ventral substantia pars reticulata $(S N r)$. In $B$ the dendritic morphology of PHA-L-labeled neurons is clearly evident. Labeled dendrites are distributed mediolaterally in the plane of the pars compacta. In $C$ the dendritic morphology of labeled SNc neurons is less clear, but neurons in the dorsal tier (SNc-d) with mediolaterally directed dendrites and neurons in the ventral tier ( $\mathrm{SNc}-\mathrm{v})$ with ventrally directed dendrites are labeled. In this case there is extensive diffuse tracer labeling, which masks a clearer view of labeled neurons. In $D$, PHA-L-labeled neurons are located mainly in the ventral tier of the SNr. A few neurons in the ventral tier of the SNc are also labeled. Scale bar, $500 \mu \mathrm{M}$.

efferents, which are all TH-negative, are distributed to the thalamus, superior colliculus, and pedunculopontine nucleus, as well as to the striatum. Labeled striatal afferents are type $\mathrm{C}$ and are distributed in the matrix (Figs. $9 B ; 10, A, A^{\prime}$ ). In this and other similar cases, as opposed to cases of injections into the ventral tegmental area, TH-positive, type C-like fibers have not been identified.

Retrorubral area. Striatal projection neurons in the retrorubral area are more scattered than are those in the pars compacta (see Fig. 1, $C, C^{\prime}$ ). PHA-L injections into this area label a relatively sparse projection to the striatum, presumably because any single injection labels a small number of striatally projecting retrorubral neurons. In the striatum PHA-L-labeled fibers express $\mathrm{TH}$ immunoreactivity, are type $\mathrm{A}$, and are distributed in the matrix compartment (Fig. 9A).

\section{Topography}

All of the above representative injections label afferents distributed throughout the rostrocaudal extent of the striatum. The dorsoventral and mediolateral distribution of afferents varies systematically with afferent origin. A summary of all cases is depicted in Figure 11.

\section{Discussion}

Previous studies of the mesostriatal system

Midbrain dopaminergic cells have classically been divided into 3 cell groups: the A10 in the ventral tegmental area, the A9 in the substantia nigra, and the A8 in the retrorubral area (Dahlström and Fuxe, 1964). Initially, these divisions were thought to reflect, in part, separate dopaminergic projections from the A10 to limbic forebrain areas and from the A9 and A8 to the striatum (Dahlström and Fuxe, 1964; Ungerstedt, 1971). The perception of the segregation of the A10 mesolimbic and A9/ A8 nigrostriatal systems was modified by Fallon and Moore (1978) and Lindvall et al. (1978), who showed that A10 neurons in the ventral tegmental area are continuous with A9 neurons in the pars compacta in providing a topographically organized projection to the forebrain. Another parcellation of midbrain

\footnotetext{
Figure 4. Color plate showing colocalization of PHA-L $(A-D)$ and TH $\left(A^{\prime}-D^{\prime}\right)$ immunoreactivity in the same sections obtained by photographing a section with a rhodamine-specific fluorescence filter to identify PHA-L immunoreactivity labeled red with GP $\alpha$ PHA-L-G $\alpha$ GP-TRITC $(A-D)$ and then with a fluorescein-specific fluorescence filter to identify TH immunoreactivity labeled green with R $\alpha \mathrm{TH}-\mathrm{G} \alpha \mathrm{R}-\mathrm{FITC}\left(A^{\prime}-D^{\prime}\right)$. In $A$, type A striatal afferents labeled with PHA-L are seen to express TH immunoreactivity in $A^{\prime}$. These fibers are distributed in the striatal matrix. In $B$, type B striatal afferents labeled with PHA-L are also seen to express TH immunoreactivity $\left(B^{\prime}\right)$. These fibers are located within a striatal patch. In $C$, type $\mathrm{C}$ striatal afferents labeled with PHA-L, with their distinctive bulbous boutons, do not express TH immunoreactivity $\left(C^{\prime}\right)$. In $D$, PHA-Llabeled striatal afferents in the olfactory tubercle are seen to express TH immunoreactivity $\left(D^{\prime}\right)$.
} 

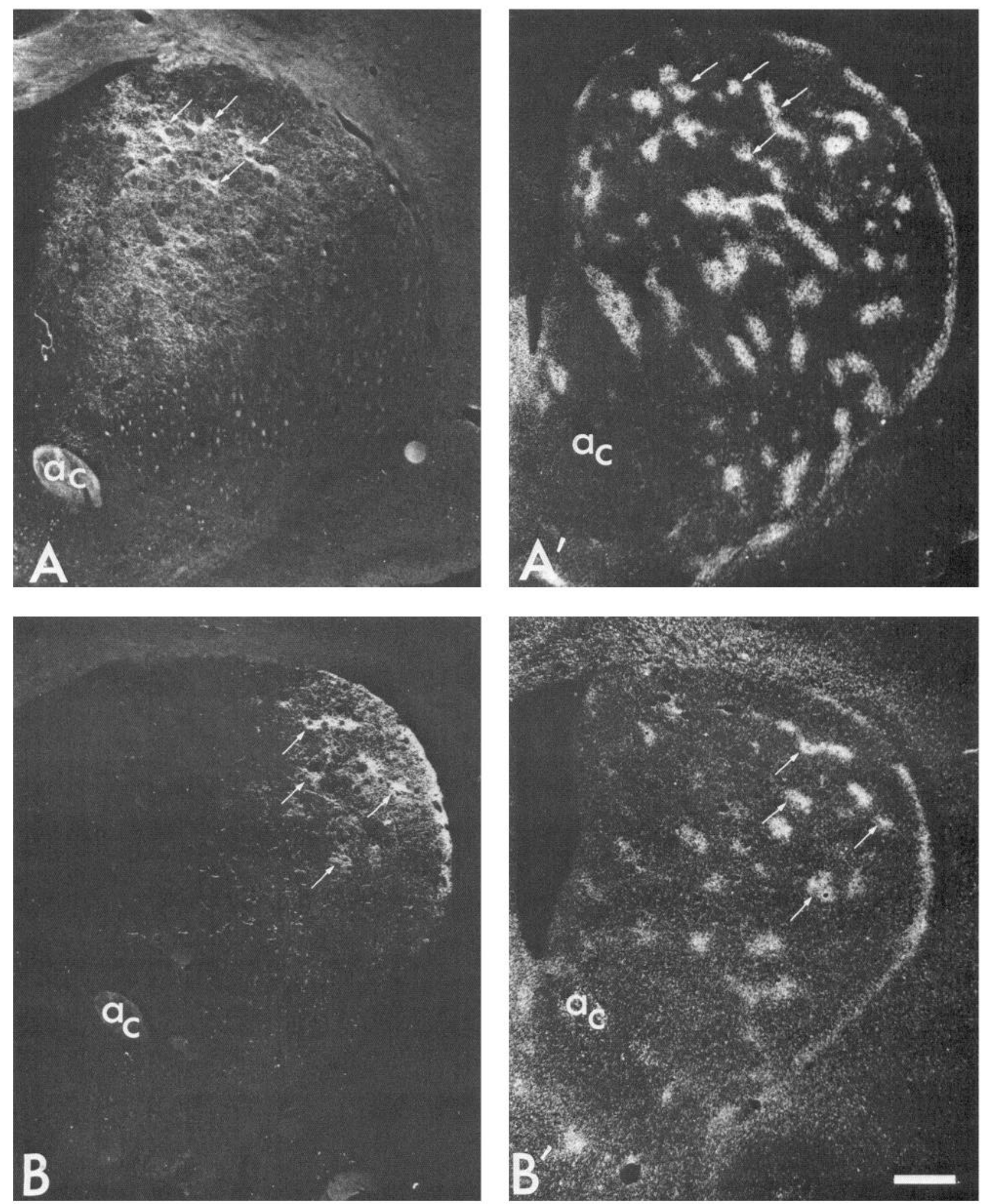

Figure 6. Adjacent sections through the striatum from 2 cases of PHA-L injections into different parts of the substantia nigra are photographed with dark-field microscopy (positive labeling is white) at low power to show the distribution of PHA-L-labeled mesostriatal afferents ( $A, B)$ and ${ }^{3} \mathrm{H}$-naloxone binding of $\mu$-opiate receptor-rich striatal patches $\left(A^{\prime}, B^{\prime}\right)$. $A$, PHA-L-labeled mesostriatal afferents from the dorsal and ventral tier of the substantia nigra pars compacta (Fig. $5 \mathrm{C}$ ) are primarily dopaminergic (fiber types A and B), distributed in both patch and matrix areas of the dorsal-central striatum (patch areas marked in $A^{\prime}$ ). In this case there appears to be a somewhat denser distribution of afferents in some patches; however, when examined at higher magnification (Fig. 7C), there is also dense labeling in the matrix. B, PHA-L-labeled nigrostriatal afferents from the ventral pars reticulata (Fig. $5 D$ ) are primarily type B dopaminergic afferents. In this case striatal patches in the dorsolateral striatum are densely innervated (patches in $B^{\prime}$ ). The paucity of afferent labeling in the matrix is shown at higher magnification in Figure $7 B$. Arrows, patches with both PHA-L-labeled afferents and ${ }^{3} \mathrm{H}$-naloxone. Scale bar, $500 \mu \mathrm{m}$. 

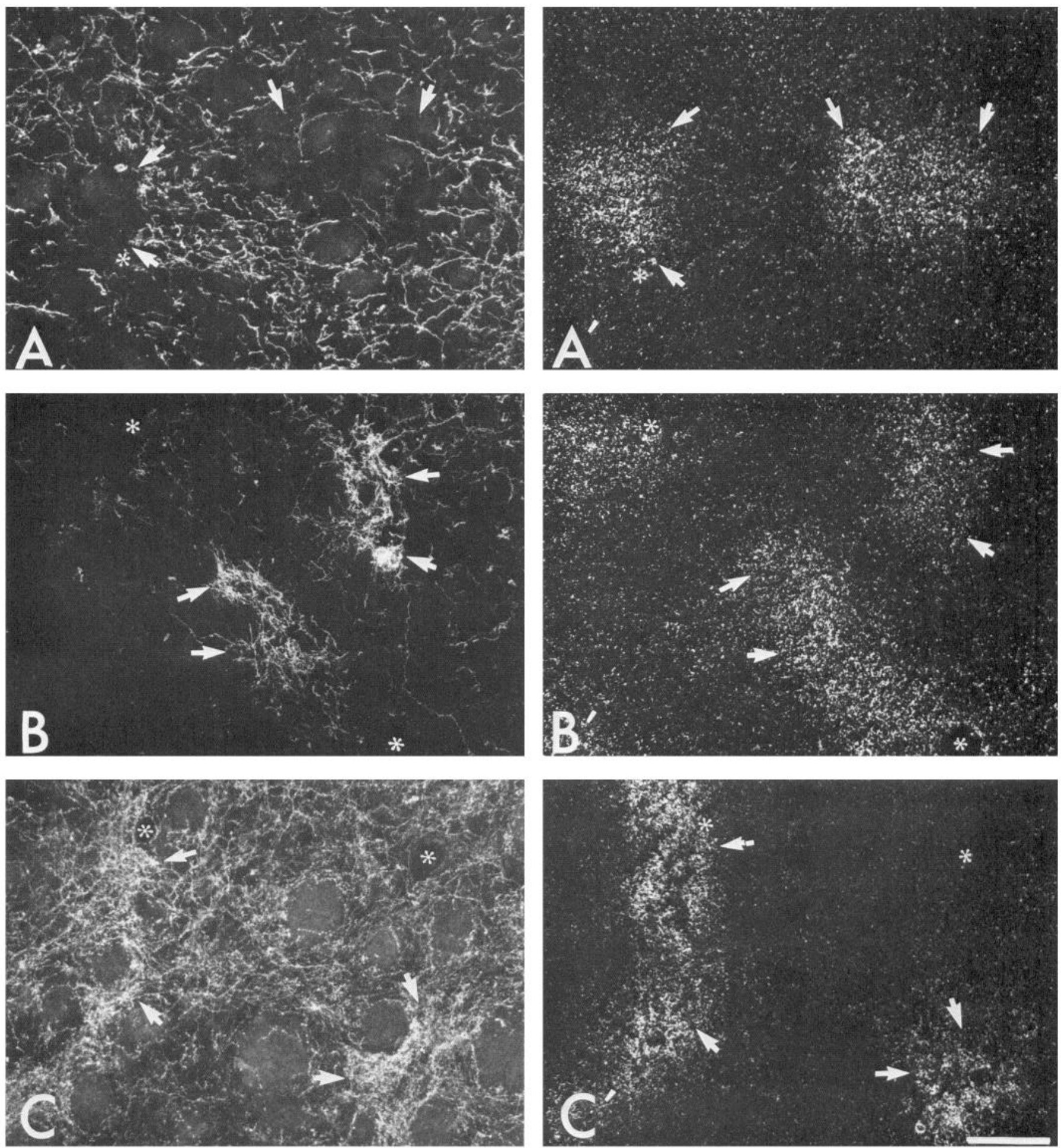

Figure 7. High-power dark-field photomicrographs (positive labeling is white) from striatal sections, such as those shown in Figure 5, show the distribution of PHA-L-labeled mesostriatal afferents $(A-C)$ as compared to the striatal compartments marked in adjacent sections with ${ }^{3} \mathrm{H}$-naloxone binding to $\mu$-opiate receptors in patches $\left(A^{\prime}-C^{\prime}\right)$. $A$, PHA-L-labeled mesostriatal afferents from the dorsal tier of the substantia nigra pars compacta (Fig. $5 B$, SNc-d) are of type A and are distributed primarily in the matrix (arrows in $A$ point to corresponding patches in $A^{\prime}$ ). $B$, Mesostriatal afferents from the substantia nigra pars reticulata (Fig. $5 D, \mathrm{SNr}$ ) are dopaminergic type $\mathrm{B}$ afferents and are distributed primarily in the striatal patches (arrows in $B$ point to corresponding patches in $B^{\prime}$ ). $C$, Mesostriatal afferents from the dorsal and ventral tier of the substantia nigra pars compacta (Fig. $5 C$, SNc-d, SNc-v) are primarily dopaminergic type A and B afferents and are distributed in both patch and matrix compartments (arrows in $C$ point to corresponding patches marked in $C^{\prime}$ ). In this case, labeled type B fibers in the patches are denser than are type $\mathrm{A}$ fibers in the matrix. However, the relative densities of labeling in the 2 compartments are more homogenous than that from cases shown in $A$ and $B$. Asterisks mark fiber bundles and blood vessels used to align adjacent sections. Scale bar, $250 \mu \mathrm{m}$. 

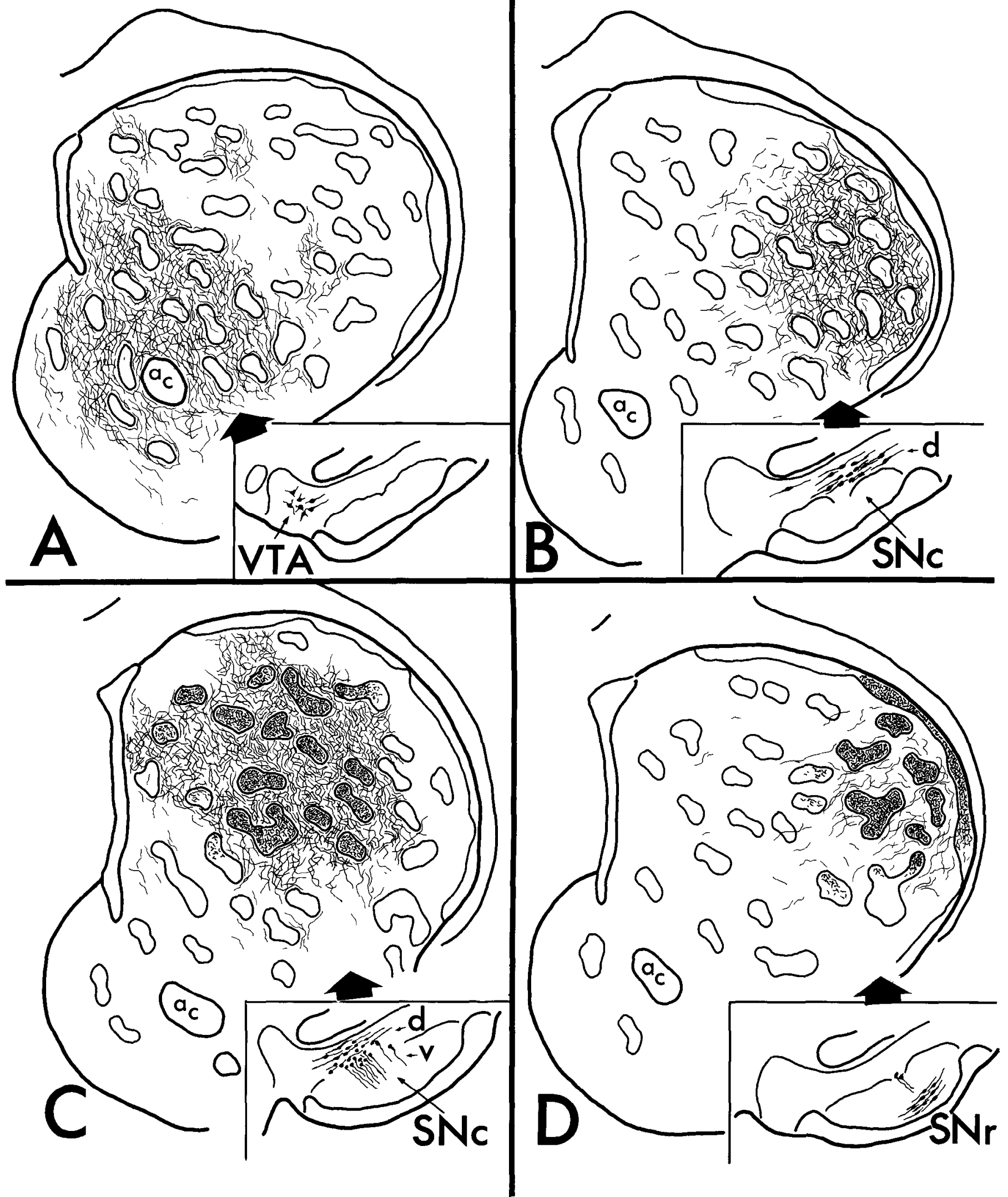

Figure 8. Diagrams of camera lucida chartings of four PHA-L injections (insets) into $(A)$ ventral tegmental area (VTA), (B) dorsal tier (d) of the substantia nigra pars compacta $(S N C),(C)$ dorsal $(d)$ and ventral $(v)$ tiers of the $S N c$, and $D)$ ventral substantia nigra pars reticulata (SNr) and resulting labeling of mesostriatal afferents charted relative to striatal patch and matrix compartments, as determined by the distribution of ${ }^{3} \mathrm{H}$ - 
dopaminergic neurons emerged, namely the existence of 2 tiers of neurons, a dorsal tier including the ventral tegmental area and dorsally situated pars compacta neurons, and a ventral tier of lateral ventral tegmental area cells and ventral pars compacta neurons (Fallon and Moore, 1978). The dendritic morphologies of the 2 tiers of pars compacta neurons are distinct; those in the dorsal tier extend dendrites mediolaterally in the plane of the nucleus (Fallon et al., 1978), whereas those in the ventral tier extend dendrites ventrally into the pars reticulata (Björklund and Lindvall, 1975). Initially, the dorsal tier neurons were thought to correspond to the mesolimbic system and the ventral tier to the nigrostriatal system. However, more recent studies show that some dopaminergic neurons provide collaterals to both limbic cortex and striatum (Fallon and Loughlin, 1982; Loughlin and Fallon, 1984; Takada and Hattori, 1986) and that both the dorsal and ventral tier systems provide inputs to the striatum (Veening et al., 1980). Our data suggest that the 2 tiers of midbrain dopaminergic neurons project differentially to the striatal patch and matrix compartments.

\section{Compartmental mesostriatal projections}

Non-dopaminergic projections to the matrix. The present results describe the distribution of nigrostriatal non-dopaminergic type $\mathrm{C}$ fibers to the striatal matrix compartment. The existence of a non-dopaminergic nigrostriatal system was previously inferred from the findings that (1) 6-OHDA fails to destroy all nigrostriatal projections (Fibiger et al., 1972), (2) glyoxylic acid-induced catecholamine fluorescence is not present in $5-10 \%$ of retrogradely labeled nigrostriatal neurons (van der Kooy et al., 1981), (3) some 5-15\% retrogradely labeled mesostriatal neurons do not express TH immunoreactivity (Swanson, 1982), and (4) nigral neurons with collaterals to the thalamus and striatum, unlikely to be dopaminergic, can be demonstrated electrophysiologically (Deniau et al., 1978). The present study uses another means of identification of non-dopaminergic nigrostriatal projections, namely, failure to express TH immunoreactivity in PHA-L-labeled fibers (Gerfen and Sawchenko, 1985). Results using this method are consistent with previous reports in that the origin of such labeled non-dopaminergic afferents is from areas previously identified as containing non-dopaminergic nigrostriatal neurons, i.e., either in the rostral mediodorsal pars reticulata or in the ventrolateral tier of the pars reticulata (van der Kooy et al., 1981; Swanson, 1982).

Mesostriatal afferents that do not display TH immunoreactivity, which we have designated type $\mathrm{C}$, are morphologically distinct from those that do. Type $\mathrm{C}$ fibers are characterized by their thickness $(0.4-0.7 \mu \mathrm{m})$, arborization pattern, and large bulbous boutons (up to $2.0 \mu \mathrm{m}$ ). The very rare labeling of type C fibers with TH immunoreactivity in the normal or partially dopamine-depleted striatum supports the suggestion that type $\mathrm{C}$ fibers are most often non-dopaminergic. Also, in electron- microscopic analysis of TH-immunoreactive striatal terminals, a comparable terminal type is not labeled (Pickel et al., 1981; Arluison et al., 1984; Freund et al., 1984). However, recently Voorn et al. (1986) have identified large boutons that contain dopamine immunoreactivity in the ventral striatum. Such fibers are occasionally labeled in the present study, and they were always found in the ventral striatum. A close examination of $\mathrm{TH}$-negative type $\mathrm{C}$ fibers in the dorsal striatum revealed that they have slightly larger boutons than the TH-positive type Clike boutons in the ventral striatum (which are similar to those in the adjacent olfactory tubercle and so may represent displaced afferents to this area).

The chemical phenotype of TH-negative type $\mathrm{C}$ fibers has not been determined. However, the report that some nigrostriatal fibers are collaterals of nigrothalamic neurons (Deniau et al., 1978), which are predominantly GABAergic, would suggest that non-dopaminergic mesostriatal afferents may also be GABAergic.

Dopaminergic afferents to the striatal matrix. Herkenham et al. (1984) have shown that the ventral tegmental area projects preferentially to the matrix compartment of the ventral striatum, including the nucleus accumbens. The present results confirm that this projection is primarily dopaminergic and suggest further that dopaminergic neurons in the dorsal tier of the substantia nigra pars compacta and in the retrorubral area provide inputs to the striatal matrix as well.

Dissociating the projections of dorsal and ventral tier pars compacta neurons is complicated by the close proximity of these 2 sets of neurons. The PHA-L method proved useful in this context because of its ability to clearly identify the neurons and their dendritic morphology, whose efferent projections are labeled. In one fortuitous case, $85 \%$ of the PHA-L-labeled neurons were in the dorsal tier of the pars compacta with dendrites distributed mediolaterally in the plane of the pars compacta (Fig. $5 B$ ). In this case the majority of labeled dopaminergic striatal afferents was distributed to the matrix compartment (Fig. $7 A$ ). In cases in which there was a more even distribution between dorsal and ventral tier pars compacta neurons labeled with PHA-L (Fig. 5C), there was also a more even distribution of labeled efferents to both patch and matrix compartments (Fig. $7 C$ ). There are a number of obvious limitations that must be considered in drawing conclusions based on these data. In the case in which the majority of labeled neurons were located in the dorsal tier, not all dorsal tier neurons were labeled. Also, we were not able to selectively label the projections of ventral tier neurons. Nonetheless, the data offer compelling evidence that dorsal tier neurons project to the matrix, and ventral tier neurons project to the patches. Data provided in the following paper (Gerfen et al., 1987), using different methods, supports this conclusion. To summarize those data, neonatal striatal 6-OHDA lesions, which selectively destroy the mesostriatal inputs to the

\footnotetext{
naloxone binding of $\mu$-opiate receptor-rich patches in adjacent sections. In these diagrams the striatal patches are outlined (see Fig. $6, A^{\prime}, B^{\prime}$ for photographs of typical labeling). In these chartings only PHA-L-labeled dopaminergic type $A$ and $B$ fiber types are depicted, and, as shown in Figure 2 and discussed in the text, type A fibers are distributed principally in the striatal matrix and type B fibers are distributed in the striatal patches. Coronal sections at roughly the same rostrocaudal level are shown for each case. $A$, PHA-L-labeled neurons in the VTA provide type A afferents to the matrix compartment in the ventral striatum. $B$, PHA-L-labeled neurons in the dorsal tier of the SNc, with mediolaterally directed dendrites, provide type $\mathrm{A}$ afferents to the matrix compartment of the dorsolateral striatum. $C$, PHA-L-labeled neurons in the dorsal $(d)$ and ventral (v) SNc provide type A afferents to the striatal matrix and type B afferents to the striatal patches. D, PHA-L-labeled dopaminergic neurons in the ventral SNr provide type B afferents to the patches of the dorsolateral striatum. A summary of labeling at other levels for all cases is provided in Figurc 11.
} 


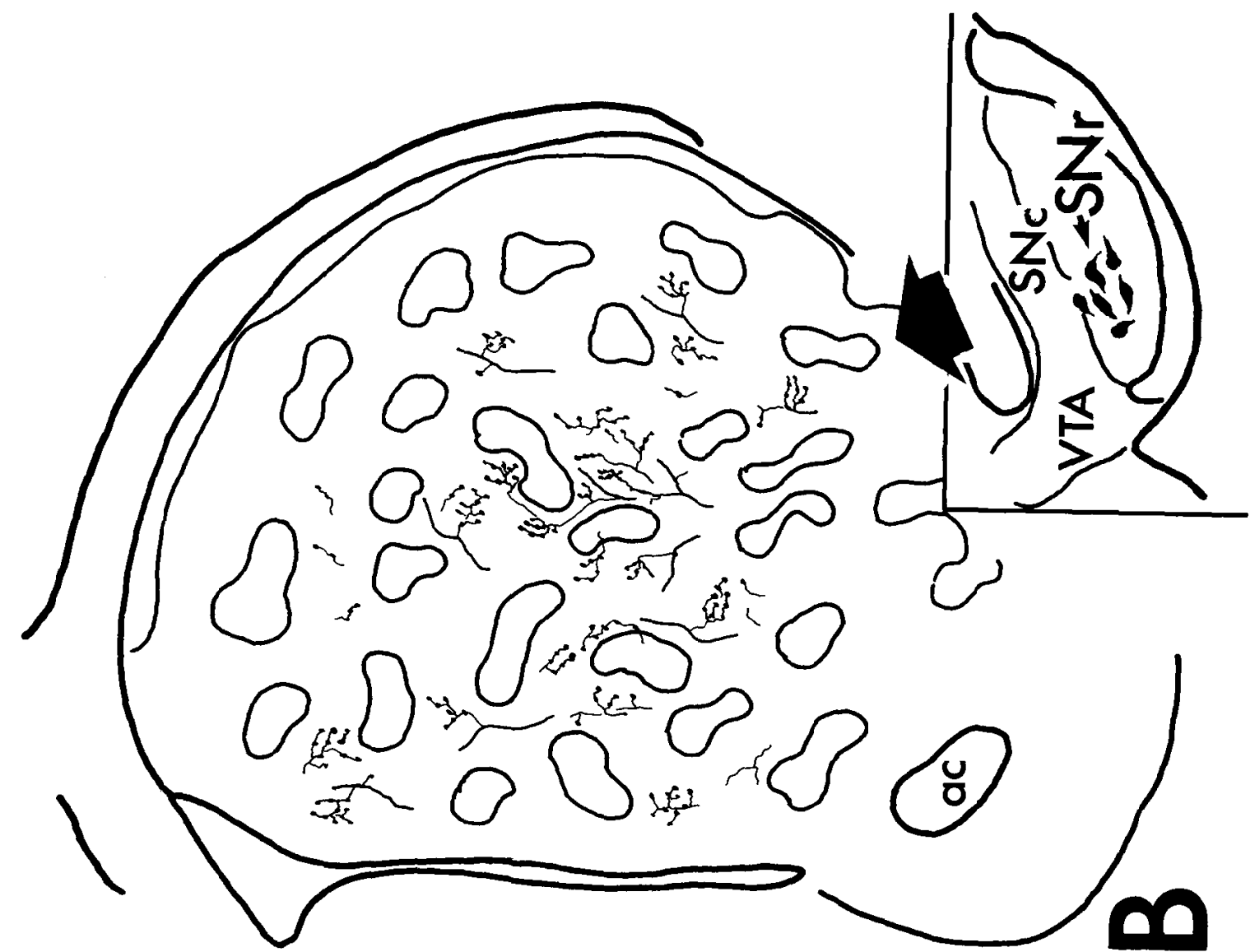

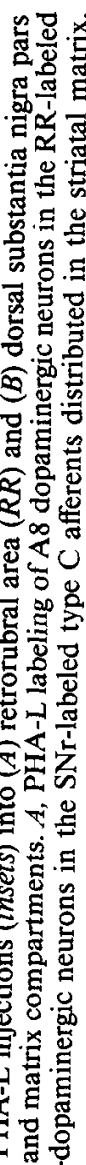

눌

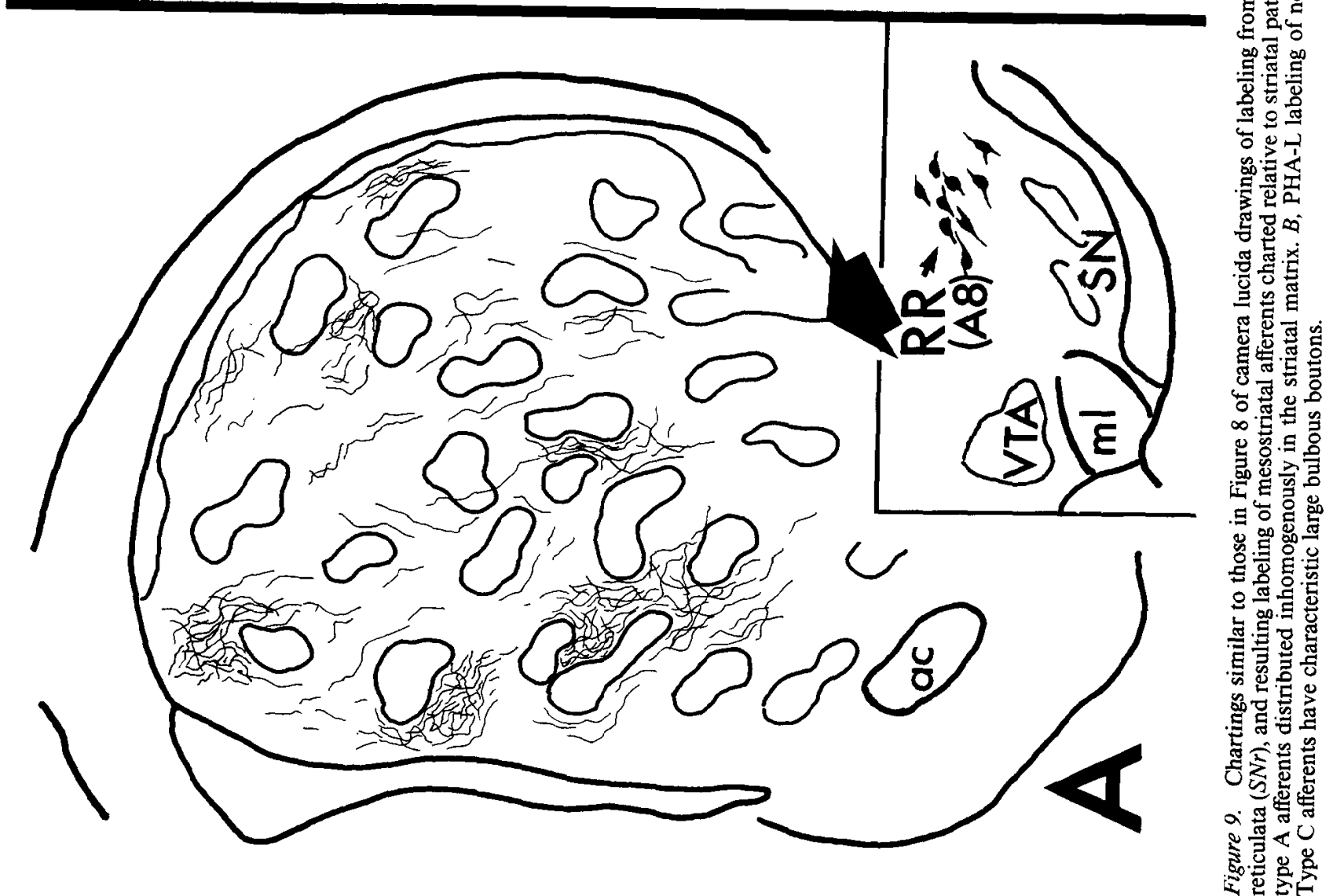



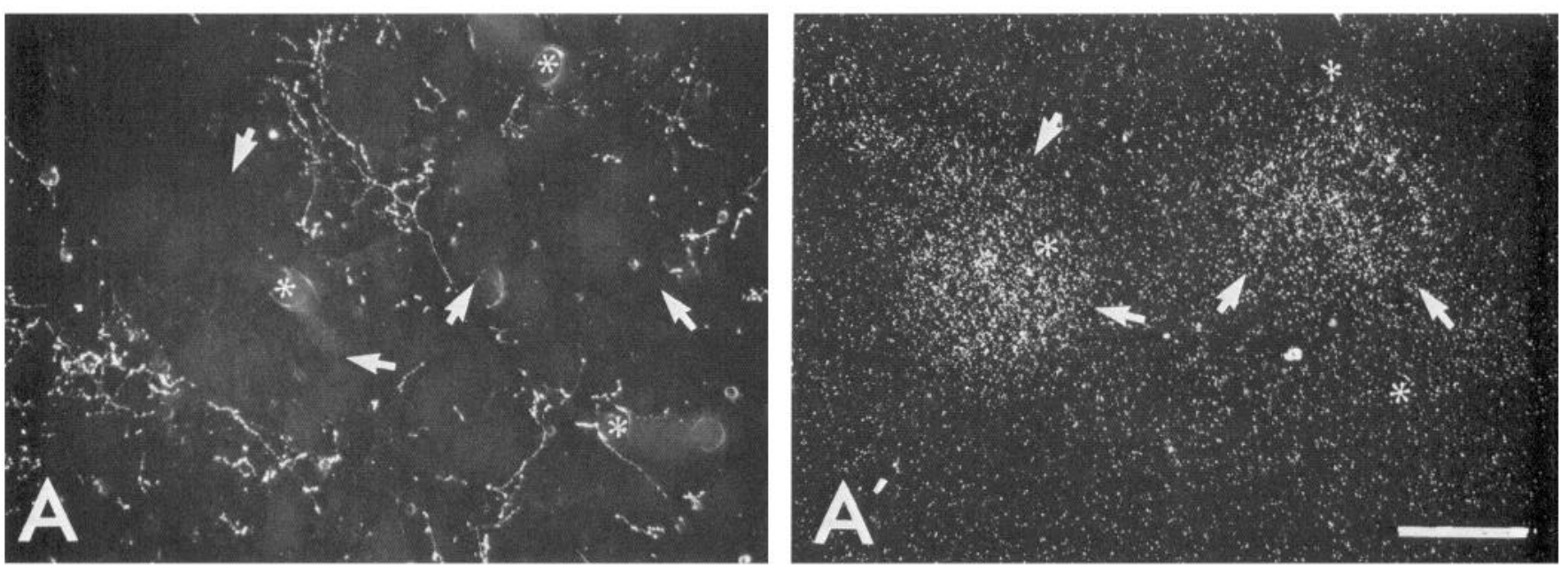

Figure 10. High-power dark-field (positive labeling is white) photomicrographs from striatal sections from the case shown in Figure $9 B$ showing the distribution of PHA-L-labeled nigrostriatal afferents $(A)$ relative to the striatal compartments marked in adjacent sections with ${ }^{3} \mathrm{H}$-naloxone binding to $\mu$-opiate receptors in patches $\left(A^{\prime}\right)$. PHA-L-labeled type C fibers $(A)$ are distributed in the matrix compartment. Arrows, area of patches in $A$ marked by ${ }^{3} \mathrm{H}$-naloxone binding in patches in $A^{\prime}$. Asterisks, blood vessels at the border of patches in the 2 sections. Scale bar, $250 \mu \mathrm{m}$.

patches while sparing the later developing inputs to the matrix, result in the select survival of dorsal pars compacta neurons with dendrites that extend in the plane of the pars compacta (Gerfen et al., 1987). Mesostriatal dopaminergic neurons projecting to the striatal matrix may also be biochemically distinguished. The distribution of such neurons is similar to that of midbrain dopamine neurons that express a $28 \mathrm{kDa}$ brain calcium binding protein (Gerfen et al., 1985, 1987).

There appears to be a rough topography of mesostriatal projections to the matrix (see Fig. 11). Ventral tegmental area inputs are directed to the nucleus accumbens and ventromedial caudate putamen; dorsal pars compacta neurons project to the dorsolateral striatum; and retrorubral projections appear to be rather diffusely distributed in the dorsal striatum. This topography is consistent with previous reports (Fallon and Moore, 1978; Beckstead et al., 1979; Veening et al., 1980). However, it is necessary to emphasize that this is only a rough topography, as there are medial and lateral pars compacta neurons which project to lateral and medial striatal areas, respectively, intermixing with those projecting topographically (van der Kooy, 1979; Parent et al., 1983). This is seen in the present material, with considerable overlap among the afferent fields from each area. A similar organization of striatonigral projections has been described (Gerfen, 1985).

Dopaminergic afferents to striatal patches. The present results suggest that dopaminergic neurons in the ventral tier of the pars compacta and most dopaminergic neurons in the pars reticulata project to the striatal patches. Because the dopaminergic neurons in the pars reticulata are segregated from other dopaminergic neurons, it was possible to obtain relatively pure injections of these neurons with PHA-L (Fig. 5D). Such cases showed clearly that dopaminergic striatal afferents provide inputs directed principally to the striatal patches (Fig. $7 B$ ). Also, as discussed above, PHA-L cases suggest that ventral tier pars compacta neurons project to the striatal patches. Selective destruction of ventral tier pars compacta neurons and pars reticulata dopaminergic neurons with neonatal striatal 6-OHDA lesions, which eliminate patch but not matrix dopaminergic afferents, supports this suggestion (see the following paper, Gerfen et al., 1987). The dendritic morphology of patch-projecting ventral tier neurons is distinctive in that they extend ventrally into the pars reticulata, as previously described by Björklund and Lindvall (1975). Similarly, the other set of patch-directed dopaminergic afferents arise from pars reticulata neurons whose dendrites also extend into the pars reticulata. The significance of this relative to the differential distribution of striatonigral afferents from the patch and matrix compartments is discussed below.

As with mesostriatal projections to the matrix, those to the patches also appear to display a rough "topography." Medial pars compacta neurons project to the more medial striatal patches, whereas the more laterally situated pars reticulata dopaminergic neurons project to lateral striatal patches. In fact, the topography of patch-directed afferents appears to be somewhat more rigid than that to the matrix. Significantly, as reported by Herkenman et al. (1984), there is a paucity of dopaminergic input to patches in the nucleus accumbens.

Morphologies of patch and matrix dopaminergic afferents. The morphologies of dopaminergic afferents in the patches and matrix are distinct (Fig. 2, $A, B$ ). Differences in the fiber morphology of patch and matrix dopaminergic afferents have been described using catecholamine histofluorescence techniques in which fibers in patches have a distinct dotted appearance, whereas those in the matrix appear more diffuse (Olson et al., 1972; Fuxe et al., 1979; Agnati et al., 1982). Although the morphological resolution afforded by TH immunohistochemical labeling is comparable to that obtained with PHA-L labeling, the density of labeled fibers has permitted only cursory descriptions of differences in patch and matrix fiber types (Agnati et al., 1982). Also, a previous electron-microscopic study by Pickel et al. (1981) describes more than one type of TH-immunoreactive fiber type, but does not examine their relative distributions in patch and matrix compartments. There appears to be some consensus now among electron-microscopic studies that striatal dopaminergic afferents make mainly symmetric synaptic contacts with the spines and dendritic shafts of medium spiny neurons (Pickel et al., 1981; Bouyer et al., 1984; Freund et al., 1984). Both Pickel et al. (1981) and Bouyer et al. (1984) suggest that there are considerable areas of apposition between nonsynaptic portions of dopaminergic afferents and other afferent fibers in the stria- 


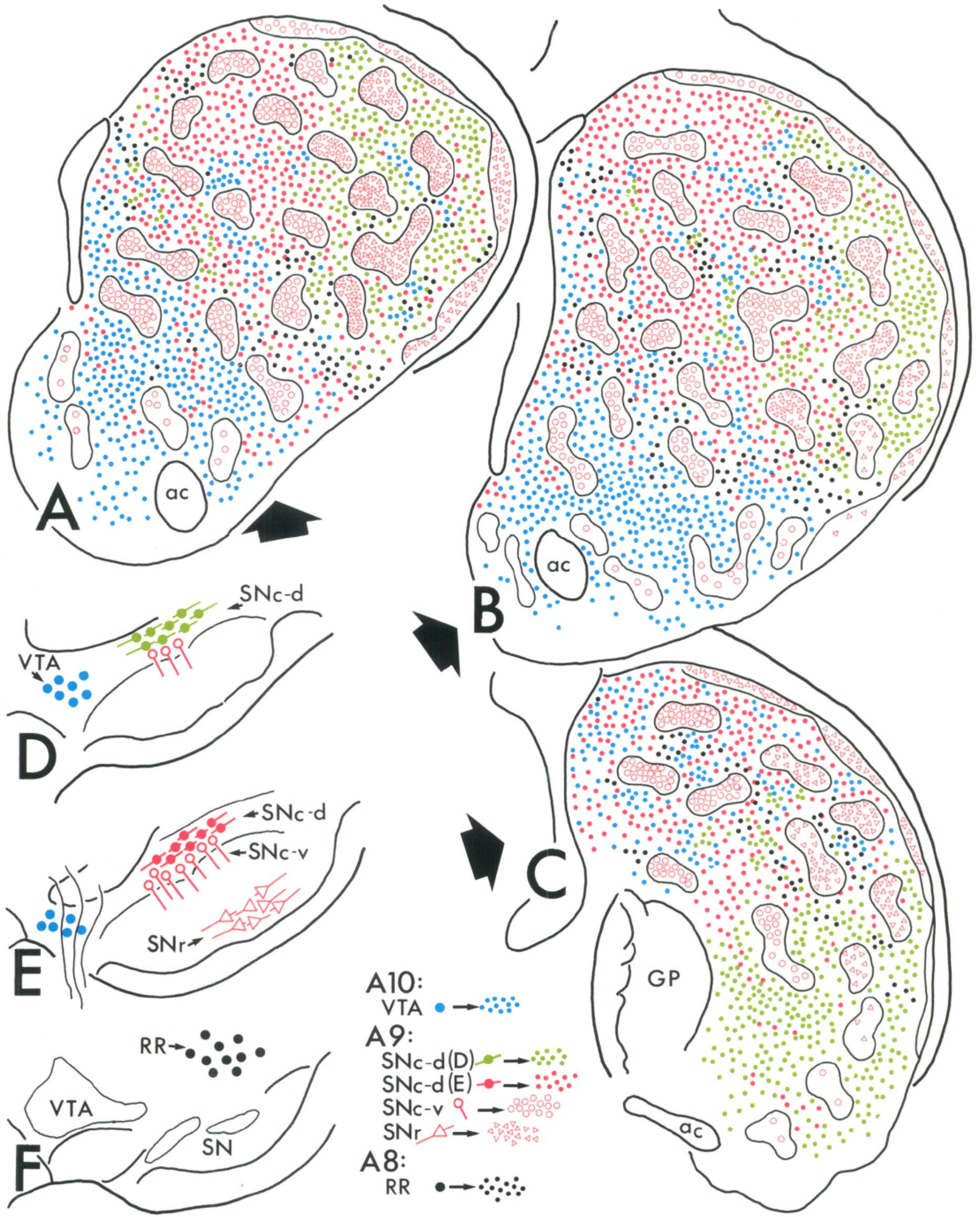

Figure 11. Diagram showing the combined distribution of mesostriatal dopaminergic afferents at rostral $(A)$, mid $(B)$, and caudal $(C)$ levels through the striatum from 6 PHA-L injections into the midbrain (from rostral to caudal, $D-F$ ). Injections into the ventral tegmental area (VTA neurons 
tum. Whether such points of contact are physiologically functional and whether the 2 types of patch and matrix afferents have more or less of these appositions awaits further analysis.

The pattern of dopaminergic fiber afferents is in marked contrast to that of the type $C$ fibers, presumed to be non-dopaminergic, which have very large bulbous boutons. Electron-microscopic analysis of these terminals is necessary to determine how they might differ from dopaminergic afferents.

\section{Relationship of mesostriatal and striatonigral systems}

Previous studies have demonstrated the existence of distinct patch and matrix striatonigral projection systems (Gerfen, 1984, 1985; Gerfen et al., 1985). Striatal neurons in the matrix project to the substantia nigra pars reticulata, except to those ventral tier parts where dopaminergic neurons are located. Conversely, striatal neurons in the patches project to the locations of the cell bodies and proximal dendrites of dopaminergic neurons in the ventral pars compacta and the ventral ticr of the pars reticulata. These systems are shown diagrammatically in Figure 12 (Striatonigral). A comparison of striatonigral organization with the distribution of mesostriatal projections to either the patches or matrix, shown in Figure 12 (Mesostriatal), suggests forms of reciprocal, as well as nonreciprocal, relationships.

The distribution of striatonigral afferents from the patches coincides with the location of the cell bodies and proximal dendrites of mesostriatal dopaminergic neurons that project back to the patches. This suggests a reciprocity between the striatonigral and mesostriatal patch systems. A mechanism for possible interaction between the patch and matrix systems is found in the dendritic configurations of patch-projecting nigral neurons; both those of the ventral tier of the pars compacta and those of the pars reticulata dopaminergic neurons extend dendrites into areas of the pars reticulata that receive striatal matrix afferents, suggesting a direct interaction between the output of the striatal matrix and the dopaminergic neurons that project back to the patches. The mechanism of this interaction may be related to the atypical function of these dendrites, which have been found to release dopamine with the possible effect of self-regulating the activity in the neurons (Björklund and Lindvall, 1975; Groves et al., 1975; Cheramy et al., 1981). The physiologic activity in these dopaminergic neurons is not always correlatcd with dendritic release of dopamine (DA). For example, substance $P$ increases neuronal discharge rates (Walker et al., 1976), but causes a decrease in dendritic DA release (Glowinski and Cheramy, 1981). Conversely, serotonin decreases neuronal discharge rates (Dray et al., 1976), but causes an increase in dendritic DA release (Glowinski and Cheramy, 1981). However, DA neuronal discharge is not always inversely related to dendritic DA release, as acetylcholine increases both neuronal discharge (Walker et al., 1976) and dendritic DA release (Glowinski and Cheramy, 1981).

In this regard Llinas et al. (1984) provide evidence to suggest that afferents to the cell bodies or to the dendrites of dopaminergic neurons may have different, partially independent, effects on the activity in nigrostriatal efferent fibers and on dendritic release of dopamine, respectively. They propose that the action potential generated in nigrostriatal efferents does not invade the dendrites, whereas hyperpolarization of the soma may initiate dendritic release of dopamine, which may also be regulated independently by dendritic afferent inputs that do not affect the activity in the cell soma. Somogyi et al. (1981b) have demonstrated with electron-microscopic techniques that the morphology of striatonigral afferents on the cell bodies of nigrostriatal neurons is distinct from that on dendrites in the pars reticulata. This raises the possibility that patch and matrix striatonigral afferents make synaptic contact with the cell body and distal dendrites, respectively, of patch-projecting nigrostriatal neurons and have different effects on these neurons.

The main sources of dopaminergic input to the striatal matrix, from the ventral tegmental area, dorsal pars compacta, and retrorubral area, would not appear to be reciprocally innervated by areas of the striatal matrix, as the striatal matrix provides inputs to the pars reticulata. Sources of inputs to dopaminergic neurons that project to the matrix are still being examined, but so far have been thought to include the nucleus accumbens, ventral pallidum, lateral hypothalamus, lateral habenula, and amygdala (Nauta et al., 1978; Herkenham and Nauta, 1979; Christoph et al., 1986). These dopaminergic neurons may also receive an input from other parts of the subtantia nigra (Grofova et al., 1982). A number of studies suggest that nigrostriatal efferent activity may be regulated, in part, by GABAergic inputs from the pars reticulata (Dray et al., 1976; Grace and Bunney, 1979; Kamata et al., 1986). Whether such regulation by pars reticulata neurons, which is suggested as being regulated by the output of the striatal matrix (Gerfen, 1984, 1985), is directed to one or both of the patch- and matrix-directed dopaminergic systems remains to be determined.

Patches may represent a dorsally dissociated component of the ventral "limbic" striatum. This suggestion is based on the observations that patches receive inputs from the prelimbic cortex (Gerfen, 1984; Donoghue and Herkenham, 1986), which itself receives inputs from limbic forebrain areas such as the amygdala (Krettek and Price, 1977). Thus, the patch compartment appears to be a system with connections more akin to the ventral "limbic" striatum (Nauta et al., 1978; Kelley et al., 1982) but that intrudes into the dorsal striatum. Despite this intrusion, the patch and matrix compartments appear to be segregated in terms of their major input-output connections due the apparent confinement of the dendrites of patch and matrix striatonigral medium spiny neurons to their respective compartments (Gerfen, 1984, 1985; Herkenham et al., 1984; Penny et al., 1984; Izzo et al., 1987), like that of the major extrinsic afferent systems from the thalamus (Herkenham and Pert, 1981; Beckstead, 1984) and cortex (Ragsdale and Graybiel, 1981; Goldman-Rakic, 1982; Gerfen, 1984; Donoghue and Herkenham, 1986). One means by which the compartments may interact was postulated to be through the somatostatin striatal interneurons, whose dendrites span both patch and matrix compartments, but whose axons

labeled blue in $D$ and $E$ ) label striatal afferents to the striatal matrix (blue dots in $A-C$ ). Injections into the rostral, dorsal tier of the substantia nigra pars compacta ( $\mathrm{SNc}-\mathrm{d}$ neurons labeled green in $D$ ) with mediolaterally directed dendrites provide afferents to the striatal matrix (green dots in $A-$ $C)$. Injections into the substantia nigra at mid-nigral levels $(E)$ label both dorsal tier SNc neurons (SNc-d neurons with mediolaterally directed dendrites marked red in $E$ ) and ventral tier SNc neurons (SNc-v neurons with ventrally directed dendrites indicated with open red triangles in $E$ provide afferents to the striatal matrix (red dots in $A-C$ ) and striatal patches (open triangles in $A-C$ ), respectively. Injections labeling dopaminergic neurons in the ventral tier of the pars reticulata ( $\mathrm{SNr}$ neurons indicated with open circles in $E$ ) provide afferents to the striatal patches (open circles in $A-C$ ). Injections into the retrorubral area (RR neurons marked black in $F$ ) provide afferents to the striatal matrix (black dots in $A-C$ ). 


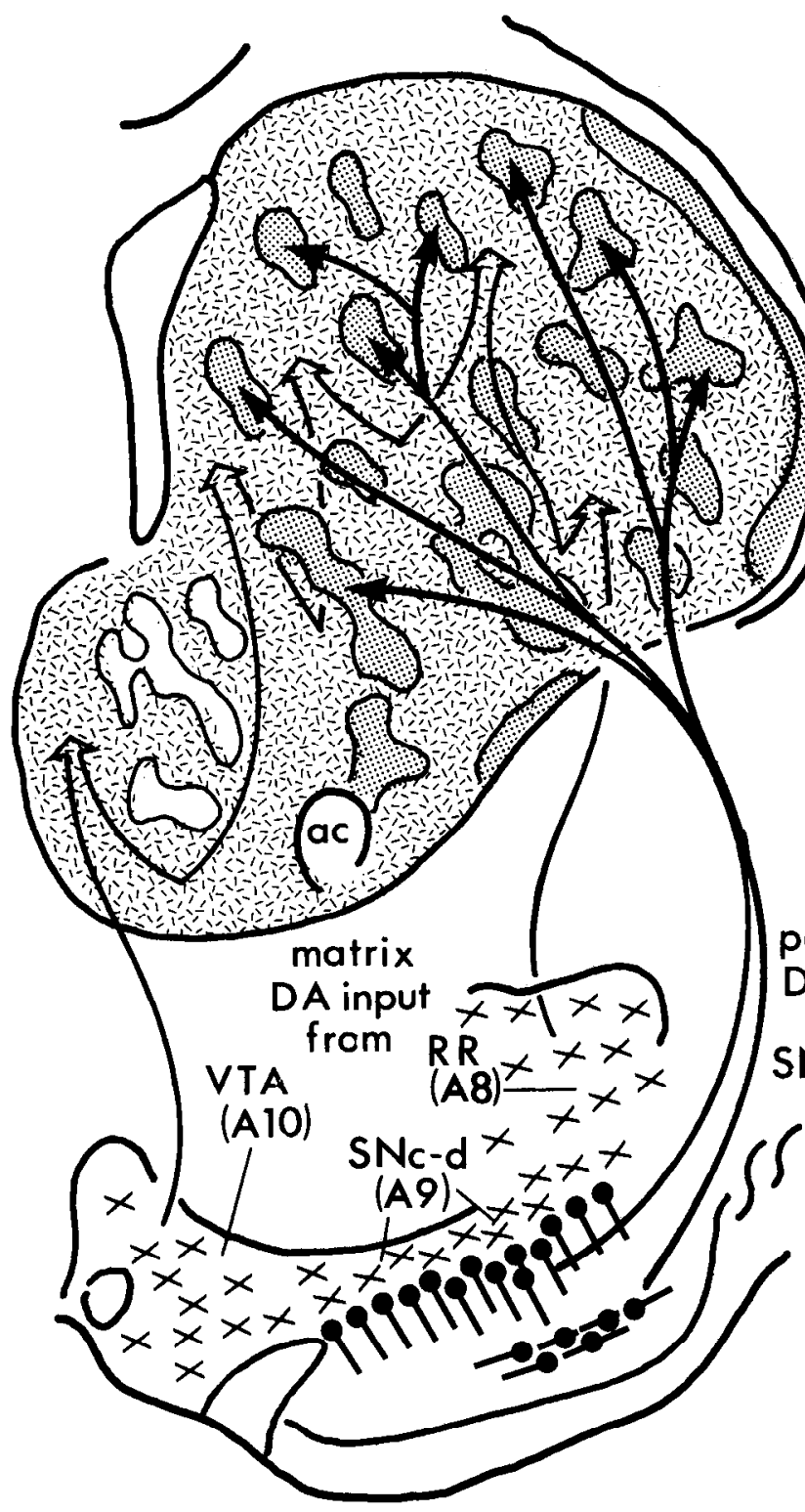

Mesostriatal

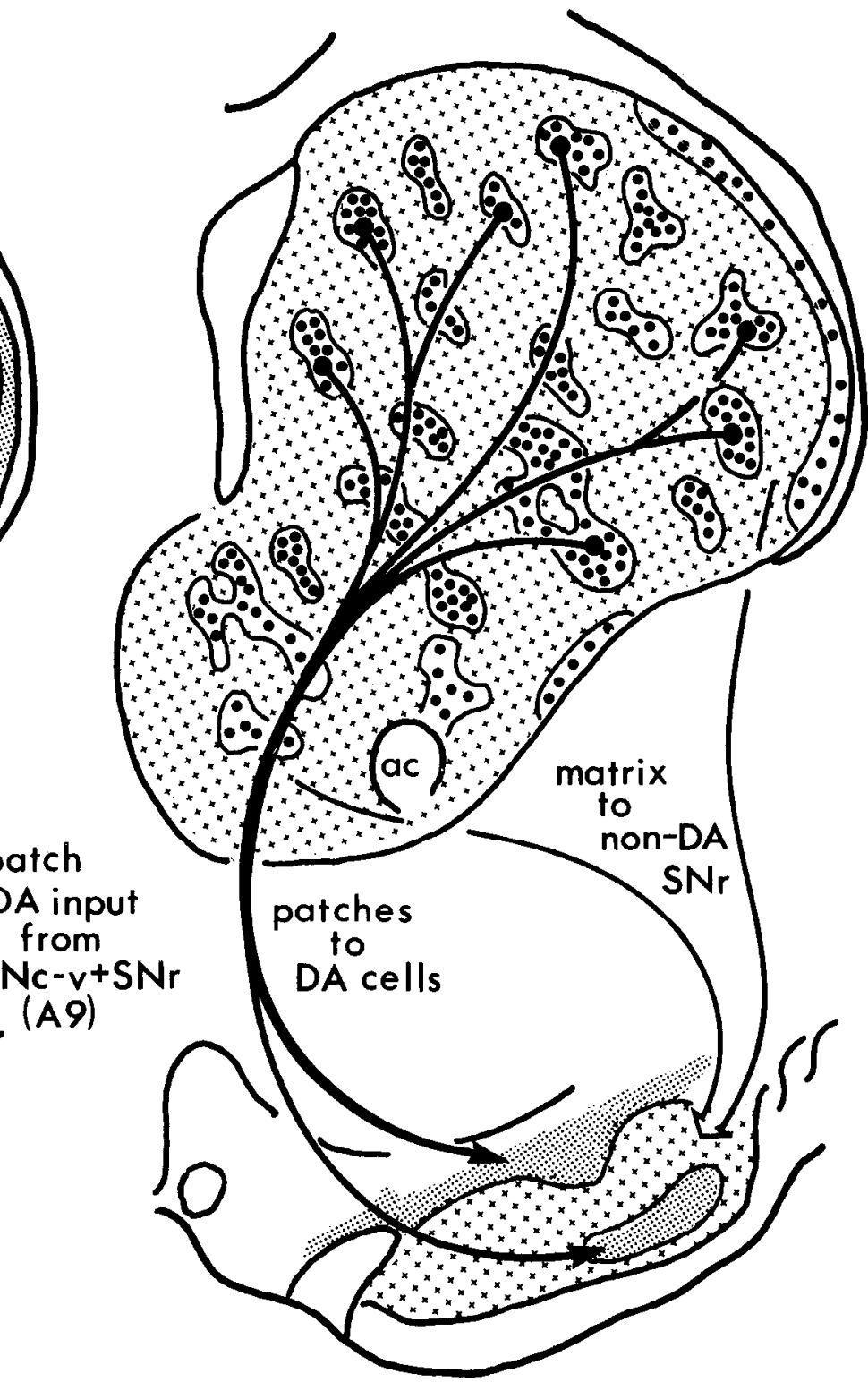

Striatonigral

Figure 12. Summary diagram of the results of these studies of the compartmental organization of the mesostriatal systems in the rat (left) compared with the compartmental organization of striatonigral systems $(B)$. Dopaminergic afferents to the striatal matrix originate from a dorsal set of midbrain neurons $(x)$ that are located in the ventral tegmental area (A10 cells in the VTA), the dorsal tier of the substantia nigra pars compacta (dorsal A9 cells in the SNc-d), and the retrorubral area (A8 cells in the RR). Dopaminergic afferents to the striatal patches originate from the ventral tier of the substantia nigra pars compacta (ventral A9 cells in the SNc-v), whose dendrites extend ventrally into the substantia nigra pars reticulata, and from the A9 DA cells located in the substantia nigra pars reticulata ( $\mathrm{SNr}$ ). Notably, afferents to the striatal patches in the nucleus accumbens are relatively sparse. The compartmental organization of the striatonigral systems is diagrammed on the right (from Gerfen, 1984, 1985; Gerfen et al., 1985). Neurons in the striatal matrix provide inputs to the substantia nigra pars reticulata that avoid the locations of DA cells in both the $\mathrm{SNc}$ and the SNr. Neurons in the striatal patches provide inputs to the location of the DA cell bodies and their proximal dendrites in the SNc-v and the SNr. However, it is possible that such neurons receive inputs from the matrix by way of contacts with their dendrites, which extend into the parts of the $\mathrm{SNr}$ that receive inputs from the matrix.

are distributed principally in the matrix (Gerfen, 1984, 1985). Another means by which the patch and matrix compartments may be interrelated is via the above-described compartmental organization of the mesostriatal systems. As discussed above, the circuitry by which this may be accomplished must be examined more closely. However, at least 2 possible mechanisms are suggested. One possibility is that inputs of the striatal matrix compartment may regulate the activity of the ventral tier mesostriatal neurons by way of inputs to their ventrally directed dendrites, which may release dopamine to autoregulate activity in the patch-directed mesostriatal system. Another possible means of interaction may occur via the regulation of patch and or matrix mesostriatal dopaminergic systems by way of collaterais of pars reticulata GABAergic neurons (Dray et al., 1976; 
Grace and Bunney, 1979; Deniau et al., 1982; Kamata et al., 1986), which receive inputs from the striatal matrix (Gerfen, $1984,1985)$. Other possible interactions between neurons within the substantia nigra, for instance between the 2 tiers of dopaminergic neurons, may provide additional interaction between the striatal compartments.

In conclusion, the present study demonstrates the organization of both dopaminergic and non-dopaminergic mesostriatal systems. Dopaminergic systems arise from dorsal and ventral tiers of midbrain neurons, whose projections target the striatal matrix and patch compartments, respectively. The manner in which this organization is related to the pharmacological and physiological differences between subsets of dopaminergic neurons, which are thought to reflect differential affects of dopaminergic systems on movement and mood (Chiodo and Bunney, 1983; Creese, 1983; White and Wang, 1983), is discussed in the following paper (Gerfen et al., 1987), in which a biochemical marker is shown to dissociate the 2 dopaminergic systems.

\section{References}

Agnati, L. F., K. Fuxe, I. Zini, L. Calza, F. Benfenati, M. Zoli, T. Hökfelt, and M. Goldstein (1982) A new approach to quantitate the density and antigen contents of high densities of transmitter-identified terminals. Immuncytochemical studies on different types of tyrosine hydroxylase immunoreactive nerve terminals in nucleus caudatus putamen of the rat. Neuroscience Lett. 32: 253-258.

Alexander, G. E., M. R. DeLong, and P. L. Strick (1986) Parallel organization of functionally segregated circuits linking basal ganglia and cortex. Ann. Rev. Neurosci. 9: 357-381.

Arluison, M., M. Dietl, and J. Thibault (1984) Ultrastructural morphology of dopaminergic nerve terminals and synapses in the striatum of the rat using tyrosine hydroxylase immunoreactivity: A topographical study. Brain Res. Bull. 13: 269-285.

Beckstead, R. M. (1984) Complementary mosaic distributions of thalamic and nigral axons in the caudate nucleus of the cat: Double anterograde labeling combining autoradiography and wheat germHRP histochemistry. Brain Res. 335: 153-159.

Beckstead, R. M., V. B. Domesick, and W. J. H. Nauta (1979) Efferent connections of the substantia ingra and ventral tegmental area in the rat. Brain Res. 175: 191-217.

Bentivoglio, M., H. G. J. M. Kuypers, C. E. Catsman-Berrevoets, H Loewe, and O. Dann (1980) Two new fluorescent retrograde neuronal tracers which are transported over long distances. Neurosci. Lett. 18: 25-30.

Bishop, G. A., H. T. Chang, and S. T. Kitai (1982) Morphological and physiological properties of neostriatal neurons: An intracellular horseradish peroxidase study in the rat. Neuroscience 7: 179-191.

Björklund, A., and O. Lindvall (1975) Dopamine in dendrites of substantia nigra neurons: Suggestions for a role in dendritic terminals. Brain Res. 83: 531-537.

Bouyer, J. J., D. H. Park, T. H. Joh, and V. M. Pickel (1984) Chemical and structural analysis of the relation between cortical inputs and tyrosine hydroxylase-containing terminals in rat neostriatum. Brain Res. 302: 267-275.

Chang, H. T., C. J. Wilson, and S. T. Kitai (1981) Single neostriatal efferent axons in the globus pallidus: A light and electron microscopic study. Science 213: 915-918.

Cheramy, A., V. Levicl and J. Glowinski (1981) Dendritic relcasc of dopamine in the substantia nigra. Nature 289: 537-542.

Chiodo, L. A., and B. S. Bunney (1983) Typical and atypical neuroleptics: Differential effects of chronic administration on the activity of $A 9$ and A 10 midbrain dopaminergic neurons. J. Neurosci. 3: $1607-$ 1619.

Christoph, G. R., R. J. Leonzio, and K. S. Wilcox (1986) Stimulation of the lateral habenula inhibits dopamine-containing neurons in the substantia nigra and ventral tegmental area of the rat. J. Neurosci. 6 : 613-619.

Creese, I. (1983) Classical and atypical antipsychotic drugs: New insights. Trends Neurosci. 6: 479-481.

Dahlström, A., and K. Fuxe (1964) Evidence for the existence of monoamine-containing neurons in the central nervous system. I. Demonstration of monoamines in the cell bodies of brain stem neurons. Acta Physiol. Scand. Suppl. 232 62: 1-31.

DeLong, M. R., and A. P. Georgopoulos (1981) Motor functions of the basal ganglia. In Handbook of Physiology, sec. 1. The Nervous System, vol. II, pt. 2, pp. 1017-1061, American Physiological Society, Bethesda, MD

Deniau, J. M., C. Hammond, A. Riszk, and J. Feger (1978) Electrophysiological properties of identified output neurons of the rat substantia nigra (pars compacta and pars reticulata): Evidence for the existence of branched neurons. Exp. Brain Res. 32: 409-422.

Deniau, J. M., S. T. Kitai, J. P. Donoghue, and I. Grofova (1982) Neural interactions in the substantia nigra pars reticulata through axon collaterals of the projection neurons. Exp. Brain Res. 47: 105113.

Donoghue, J. P., and M. Herkenham (1986) Neostriatal projections from individual cortical fields conform to histochemically distinct striatal compartments in the rat. Brain Res. 365: 397-403.

Dray, S., T. J. Gonye, N. R. Oakley, and T. Tanner (1976) Evidence for the existence of a raphe projection to the substantia nigra in rats. Brain Res. 113: 45-57.

Fallon, J. H., and S. E. Loughlin (1982) Monoamine innervation of the forebrain: Collateralization. Brain Res. Bull. 9: 295-307.

Fallon, J. H., and R. Y. Moore (1978) Catecholamine innervation of the basal forebrain. IV. Topography of the dopamine projection to the basal forebrain and neostriatum. J. Comp. Neurol. 180: 545-580.

Fallon, J. H., J. N. Riley, and R. Y. Moore (1978) Substantia nigra dopamine neurons: Separate populations project to neostriatum and allocortex. Neurosci. Lett. 7: 157-162.

Fibiger, H. C., and A. G. Phillips (1986) Reward, motivation, cognition: Psychobiology of mesotelencephalic dopamine systems. In Handbook of Physiology, The Nervous System, vol. IV: Intrinsic Regulatory Systems of the Brain, F. E. Bloom, ed., pp. 647-675, American Physiological Society, Baltimore, MD.

Fibiger, H. C., R. E. Pudritz, P. L. McGeer, and E. G. McGeer (1972) Axonal transport in nigro-striatal and nigro-thalamic neurons: Effects of medial forebrain bundle lesions and 6-hydroxydopamine. J. Neurochem. 19: 1697-1708.

Freund, T. F., J. F. Powell, and A. D. Smith (1984) Tyrosine hydroxylase-immunoreactive boutons in synaptic contact with identified striatonigral neurons, with particular reference to dendritic spines. Neuroscience 13: 1189-1215.

Fuxe, K., K. Andersson, R. Schwarz, L. F. Agnati, M. Perezde la Mara, T. Hökfelt, M. Goldstein, L. Ferland, L. Possani, and R. Tapia (1979) Studies on different types of dopamine nerve terminals in the forebrain and their possible interactions with hormones and with neurons containing GABA, glutamate, and opioid peptides. In Advances in Neurology, vol. 24, L. J. Poirier, T. L. Sourkes, and P. J. Bedard eds., pp. 199-215, Raven, New York.

Gerfen, C. R. (1984) The neostriatal mosaic: Compartmentalization of corticostriatal input and striatonigral output systems. Nature 311 : $461-464$.

Gerfen, C. R. (1985) The neostriatal mosaic: I. Compartmental organization of projections from the striatum to the substantia nigra in the rat. J. Comp. Neurol. 236: 454-476.

Gerfen, C. R. (1986) The developmental and biochemical basis of dual "patch" and "matrix" nigrostriatal systems in the rat. Soc. Neurosci. Abstr. 12: 1327.

Gerfen, C. R., and P. E. Sawchenko (1984) An anterograde neuroanatomical tracing method that shows the detailed morphology of neurons, their axons and terminals: Immunohistochemical localization of an axonally transported plant lectin, Phaseolus vulgaris-leucoagglutinin (PHA-L). Brain Res. 290: 219-238.

Gerfen, C. R., and P. E. Sawchenko (1985) A method for anterograde axonal tracing of chemically specified circuits in the central nervous system: Combined Phaseolus vulgaris-leucoagglutinin (PHA-L) tract tracing and immunohistochemistry. Brain Res. 343: 144-150.

Gerfen, C. R., K. G. Baimbridge, and J. J. Miller (1985) The neostriatal mosaic: Compartmental distribution of calcium binding protein and parvalbumin in the basal ganglia of the rat and monkey. Proc. Natl. Acad. Sci. USA 82: 8780-8784.

Gerfen, C. R., K. G. Baimbridge, and J. Thibault (1987) The neostriatal mosaic: III. Biochemical and developmental dissociation of patch-matrix nigrostriatal systems. J. Neurosci. 7: 3935-3944.

Glowinski, J., and A. Cheramy (1981) Dendritic release of dopamine; its role in the substantia nigra. In Chemical Neurotransmission: 75 
Years, L. Stjarne, P. Hedquist, H. Lagercrantz, and A. Wennmalm, eds., pp. 285-299, Academic, New York.

Goldman-Rakic, P. S. (1982) Cytoarchitectonic heterogeneity of the primate neostriatum: Subdivision into island and matrix cellular compartments. J. Comp. Neurol. 205: 398-413.

Grace, A. A., and B. S. Bunney (1979) Paradoxical excitation of nigral dopaminergic cells: Indirect mediation through reticulata inhibitory neurons. Eur. J. Pharmacol. 59: 211-218.

Graybiel, A. M., and C. W. Ragsdale, Jr. (1978) Histochemically distinct compartments in the striatum of human, monkey and cat demonstrated by acetylcholinesterase staining. Proc. Natl. Acad. Sci. USA 75: 5723-5726.

Grofova, I. (1975) The identification of striatal and pallidal neurons projecting to substantia nigra. An experimental study by means of retrograde axonal transport of horseradish peroxidase. Brain Res. 91 286-291.

Grofova, I., J. M. Deniau, and S. T. Kitai (1982) Morphology of the substantia nigra pars reticulata projection neurons intracellularly labclcd with HRP. J. Comp. Neurol. 208: 352-368.

Groves, P. M., C. J. Wilson, S. J. Young, and G. V. Rebec (1975) Selfinhibition by dopaminergic neurons. Science 190: 522-529.

Heimer, L., and R. D. Wilson (1975) The subcortical projections to the allocortex: Similarities in the neural associations of the hippocampus, the piriform cortex and the neocortex. In Golgi Centennial Symposium, M. Santini, ed., pp. 177-193, Raven, New York.

Herkenham, M., and W. J. H. Nauta (1979) Efferent connections of the habenular nuclei in the rat. J. Comp. Neurol. 187: 19-48.

Herkenham, M., and C. B. Pert (1981) Mosaic distribution of opiate receptors, parafascicular projections and acetylcholinesterase in rat striatum. Nature 291: 415-418.

Herkenham, M., and C. B. Pert (1982) Light microscopic localization of brain opiate receptors: A general autoradiographic method which preserves tissue quality. J. Neurosci. 2: 1129-1149.

Herkenham, M., S. Moon-Edley, and J. Stuart (1984) Cell clusters in the nucleus accumbens of the rat, and the mosaic relationship of opiate receptors, acetylcholinesterase and subcortical afferent terminations. Neuroscience 11: 561-593.

Hornykiewicz, O. (1966) Dopamine (3-hydroxytryptamine) and brain function. Pharmacol. Rev. 18: 925-964.

Hsu, S. M., L. Raine, and H. Fanger (1981) The use of avidin-biotin peroxidase complex $(\mathrm{ABC})$ in immunoperoxidase techniques: $A$ comparison between $\mathrm{ABC}$ and unlabeled antibody (PAP) procedures. J. Histochem. Cytochem. 29: 577-580.

Iz7o, P. N., A. M. Graybiel, and J. P. Bolam (1987) Characterization of substance P-and [met]enkephalin-immunoreactive neurons in the caudate nucleus of cat and ferret by a single section Golgi procedure. Neuroscience (in press).

Kamata, K., A. Sugimoto, and T. Kameyama (1986) Effect of chronic haloperidol on dopamine release following microinjection of GABA into the substantia nigra zona reticulata in the rat. Brain Res. 380 . $1-6$

Kelley, A. E., V. B. Domesick, and W. J. H. Nauta (1982) The amygdalostriatal projection in the rat-an anatomical study by anterograde and retrograde tracing methods. Neuroscience 7: 615-630.

Kemp, J. M., and T. P. S. Powell (1971) The structure of the caudate nucleus of the cat: Light and electron microscopy. Phil. Trans. R. Soc. Lond. [Biol.] 262: 383-401.

Krettek, J. E., and J. L. Price (1977) Projections from the amygdaloid complex to the cerebral cortex and thalamus in the rat and cat. J. Comp. Neurol. 172: 687-722.

Lindvall, O., A. Björklund, and I. Divac (1978) Organization of catecholamine neurons projecting to the frontal cortex in the rat. Brain Res. 142: 1-24.

Llinas, R., S. A. Greenfield, and II. Jahnsen (1984) Electrophysiology of pars compacta cells in the in vitro substantia nigra-a possible mechanism for dendritic release. Brain Res. 294: 127-132.

Loughlin, S. E., and J. H. Fallon (1984) Substantia nigra and ventral tegmental area projections to cortex: Topography and collateralization. Neuroscience 11: 425-435.

Moon-Edley, S., and M. Herkenham (1984) Heterogenous dopaminergic projection to the neostriatum of the rat: Nuclei of origin dictate relationship to opiate receptor patches. Anat. Rec. 208: 120A

Nauta, W. J. H., G. P. Smith, R. L. M. Faull, and V. B. Domesick (1978) Efferent connections and nigral afferents of the nucleus accumbens septi in the rat. Neuroscience 3: 385-401.

Olson, L., A. Seiger, and K. Fuxe (1972) Heterogeneity of striatal and limbic dopamine innervation: Highly fluorescent islands in developing and adult rats. Brain Res. 44: 283-288.

Parent, A., A. Mackey, and L. DeBellefeuille (1983) The subcortical afferents to caudate nucleus and putamen in primate: A fluorescence retrograde double labcling study. Ncuroscience 10: 1137-1150.

Penney, J. B., Jr., and A. B. Young (1983) Speculations on the functional anatomy of basal ganglia disorders. Annu. Rev. Neurosci. 6: 73-94.

Penny, G. R., C. J. Wilson, and S. T. Kitai (1984) The influence of neostriatal patch and matrix compartments on the dendritic geometry of spiny projection neurons in the rat as revealed by intracellular labeling with HRP combined with immunocytochemistry. Soc. Neurosci. Abstr. 10: 514.

Pert, C. B., M. J. Kuhar, and S. H. Snyder (1976) Opiate receptor: Autoradiographic localization in rat brain. Proc. Natl. Acad. Sci. USA 73: 3729-3733

Pickel, V. M., S. C. Beckley, T. H. Joh, and D. J. Reis (1981) Ultrastructural immunocytochemical localization of tyrosine hydroxylase in the rat neostriatum. Brain Res. 225: 373-385.

Ragsdale, C. W., Jr., and A. M. Graybiel (1981) The fronto-striatal projection in the cat and monkey and its relationship to inhomogeneities established by acetylcholinesterase histochemistry. Brain Res. 208: 259-266.

Sawchenko, P. E., and L. W. Swanson (1981) A method for tracing biochemically defined pathways in the central nervous system using combined fluorescence retrograde transport and immunohistochemical techniques. Brain Res. 210: 31-51.

Somogyi, P., and A. D. Smith (1979) Projection of neostriatal spiny neurons to the substantia nigra. Application of a combined Golgistaining and horseradish peroxidase transport procedure at both light and electron microscopic levels. Brain Res. 178: 3-15.

Somogyi, P., J. P. Bolam, and A. D. Smith (1981a) Monosynaptic cortical input and local axon collaterals of identified striatonigral neurons. A light and electron microscopic study using the Golgiperoxidase transport-degeneration procedure. J. Comp. Neurol. 195: $567-584$.

Somogyi, P., J. P. Bolam, S. Totterdell, and A. D. Smith (1981b) Monosynaptic input from the nucleus accumbens-ventral striatum region to retrogradely labeled nigrostriatal neurons. Brain Res. 217: 245-263.

Stevens, J. R. (1973) An anatomy of schizophrenia? Arch. Gen. Psychiatry $29: 177-189$.

Swanson, L. W. (1982) The projections of the ventral tegmental area and adjacent regions: $A$ combined fluorescent retrograde tracer and immunofluorescence study in the rat. Brain Res. Bull. 9: 321-353.

Takada, M., and T. Hattori (1986) Collateral projections from the substantia nigra to the cingulate cortex and striatum in the rat. Brain Res. 380: 331-335.

Ungerstedt, U. (1971) Stereotaxic mapping of monoamine pathways in the rat brain. Acta Physiol. Scand. (Suppl.) 367: 1-48.

van der Kooy, D. (1979) The organization of thalamic, nigral and raphe cells projecting to the medial vs lateral caudate putamen in rat. A fluorescent retrograde double labeling study. Brain Res. 169: 381387.

van der Kooy, D., D. V. Coscina, and T. Hattori (1981) Is there a non-dopaminergic nigrostriatal pathway? Neuroscience $6: 345-357$.

Veening, J. G., F. M. Corneilssen, and P. A. J. M. Lieven (1980) The topical organization of the afferents to the caudate putamen of the rat. A horseradish peroxidase study. Neuroscience 5: 1253-1268.

Voorn, P., B. Jorritsma-Byham, C. Van Dijk, and R. M. Buijs (1986) The dopaminergic innervation of the ventral striatum in the rat: A light- and electron-microscopical study with antibodies against dopamine. J. Comp. Neurol. 251: 84-99.

Walkcr, R. J., J. A. Kcmp, H. Yajima, K. Kitagawa, and G. N. Woodruff (1976) The action of substance $P$ on mesencephalic reticular and substantia nigra neurons in the rat. Experientia 32: 214-215.

White, F. J., and R. Y. Wang (1983) Differential effects of classical and atypical antipsychotic drugs on $\mathrm{A} 9$ and $\mathrm{A} 10$ dopamine neurons. Science 221: 1054-1057.

Wilson, C. J., and P. M. Groves (1980) Fine structure and synaptic connections of the common spiny neuron of the rat neostriatum: A study employing intracellular injection of horseradish peroxidase. J. Comp. Neurol. 194: 599-615.

Wright, A. K., and G. W. Arbuthnott (1981) The pattern of innervation of the corpus striatum by the substantia nigra. Neuroscience 6: 20632067. 